\title{
Consistent Proportional Trade-offs in Data Envelopment Analysis
}

\author{
Fatemeh Boloori \\ Institute for Management and Planning Studies (IMPS), Department of Economic Systems and Planning, \\ Tehran, Iran. \\ Iran University of Science and Technology, Department of Mathematics, Tehran, Iran \\ E-mail: f.boloori@gmail.com \\ Mohsen Afsharian (corresponding author) \\ Technische Universität Braunschweig, Department of Business Sciences, Braunschweig, Germany \\ E-mail: m.afsharian@tu-bs.de \\ Phone: +495313913606 \\ Fax: +495313918121
}

\begin{abstract}
Proportional trade-offs - as an enhanced form of the conventional absolute trade-offs - have recently been proposed as a method which can be used to incorporate prior views or information regarding the assessment of decision making units (DMUs) into relative efficiency measurement systems by Data Envelopment Analysis (DEA). A proportional trade-off is defined as a percentage change of the level of inputs/outputs so that the corresponding restriction is adapted with respect to the volume of the inputs and outputs of the DMUs in the analysis. It is well-known that the incorporation of trade-offs either in an absolute form or proportional form may lead in certain cases to serious problems such as infinity or even negative efficiency scores in the results. This phenomenon is often interpreted as a result of defining the set of trade-offs carelessly by the analyst. In this paper we show that this may not always be the case. The existing framework by which the trade-offs are combined mathematically to build a corresponding production technology may cause a problem rather than the definition of the trade-offs. We therefore develop analytical criteria and formulate computational methods that allow us to identify the abovementioned problematic situations and test if all proportional trade-offs are consistent so that they can be applied simultaneously. We then propose a novel framework for aggregating local sets of trade-offs, which can be combined mathematically. The respective computational procedure is shown to be effectively done by a suggested algorithm. We also illustrate how the efficiency can be measured against an overall technology, which is formed by the union of these local sets. An empirical illustration in the context of engineering schools will be presented to explain the properties and features of the suggested approach.
\end{abstract}

Keywords: Data envelopment analysis; value judgment; proportional trade-offs; consistency. 


\section{Introduction}

Weight restrictions have been the most commonly used ways to incorporate prior views or information regarding the assessment of efficiency of decision making units (DMUs) into relative efficiency measurement systems by Data Envelopment Analysis (DEA) (see, e.g., Jahanshahloo \& Soleimani-Damaneh, 2005; Bernroider \& Stix, 2007; Hatami-Marbini et al. 2015a). Different forms of weight restrictions can impose restrictions on the relative importance of input-output factors (i.e. value judgments) in order to ensure that the results are not counterintuitive (Podinovski \& Thanassoulis, 2007). Applying weight restrictions also typically improves the discrimination of the DEA models (see, e.g., Allen et al., 1997; Angulo-Meza \& Estelita Lins, 2002; Cook, Tone, \& Zhu, 2014; Joro \& Korhonen, 2015). An interesting classification of weight restrictions into different types can be found in Allen et al. (1997) and Thanassoulis, Portela and Allen, (2004).

Homogeneous weight restrictions, in particular those which restrict the ratio of weights, have often been recognized as the most promising type of weight restrictions in DEA applications (see, e.g., Podinovski \& Athanassopoulos, 1998; Khalili et al., 2010). The reason is that - in contrast to, e.g., non-homogeneous weight restrictions - DEA models with homogeneous ones produce optimal weights that still represent a DMU under evaluation in its best possible light, i.e. efficiency scores are not underestimated. Despite all benefits of using such weight restrictions, the meaning of radial projection of DEA models may not be straightforwardly obvious and might, in certain cases, cause confusion over the interpretation of the resulting efficiency scores. As a solution to this problem, Podinovski (2004a) suggested working with the dual form of these weight restrictions, which were called by the author "production trade-offs". While weight restrictions are imposed to multipliers of DEA models, production trade-offs, as their dual forms - which impose simultaneous changes of the level of inputs/outputs - are incorporated directly into the envelopment DEA models.

According to Podinovski (2004a), a certain trade-off between inputs and/or outputs can be applied if "all" involved DMUs accept it as an additional value judgement in the analysis. However, conventional trade-offs are defined in an "absolute" form, which does not account for 
the size of the DMUs. This can be seen by many DMUs as counter-intuitive and as a source of inequity in measuring their efficiency. It may be argued that the definition of a particular tradeoff should be adapted with respect to the volume of their inputs and outputs. Only with this, DMUs with varying sizes may accept such a trade-off to be imposed to the way their relative efficiency is measured. This drawback of absolute trade-offs also leads to obtaining DEA models which are not units invariant either. This means that - unlike in standard DEA models efficiency scores will depend on the units in which the vectors of absolute trade-offs are defined.

Against this background, Alirezaee and Boloori (2012) have recently proposed a method by which one can impose trade-offs in a "proportional way". In their approach, a trade-off is defined as a "percentage" change of the level of inputs/outputs rather than an absolute one as outlined above. This will not only solve the aforementioned problems, but also provides a number of other advantages. Their approach, e.g., allows incorporating a standard returns to scale (such as constant and variable returns to scale: CRS and VRS, respectively) into DEA models as a particular proportional trade-off. With respect to this property, one can also impose any hybrid returns to scale, which combines CRS and VRS in a desired form. The hybrid returns to scale proposed first by Podinovski (2004b, 2009) - can be used in situations, where the assumption of CRS is required with respect to a selected set of inputs and outputs, preserving the VRS assumption for the remaining factors (see also Afsharian, Ahn, \& Alirezaee, 2015).

It is well-known that the incorporation of trade-offs in any form (either in an absolute form or in an enhanced proportional form) may lead in certain cases to serious problems such as infinity or even negative efficiency scores in the results (see Podinovski \& Bouzdine-Chameeva, 2013; 2015). This phenomenon may be interpreted as a result of defining the set of trade-offs "carelessly" by the analyst. As it will be shown later, this may not always be the case. It can be argued that even if imposing all trade-offs "collectively" results in, e.g., an infinite expansion of the PPS, this does not always imply that the trade-offs have been defined carelessly. There are situations in which all carefully-defined trade-offs can separately be applied without any problem. However, mathematical issues such as infinity or negative scores occur only when the trade-offs are applied all together simultaneously. Therefore, what can best be said here is straightforward: there exist a few trade-offs which cannot be aggregated in "a conventional way" 
to expand the PPS. In other words, the existing framework by which the "trade-offs are combined mathematically" to form the PPS may cause a problem rather than the definition of the trade-offs.

Under these circumstances, after some preliminaries and technical background in Section 2, we investigate in Section 3 situations in which imposing a particular trade-off can result in problems such as free production or infeasible expansion of the PPS. Furthermore, mathematical programming models are formulated to test if "all trade-offs" are consistent so that they can be applied simultaneously. In Section 4.1, we revisit the conventional approach of combining tradeoffs. We identify shortcomings in this approach and use it as a starting point to develop a new approach of aggregating trade-offs, which avoids the above mentioned problems. As will be shown in Section 4.2, our approach requires finding maximal consistent subsets of trade-offs to create local PPSs. We hence propose an effective algorithm by which these sets can be identified. Section 4.3 illustrates how the efficiency can be measured against a PPS which is formed by the union of the local PPSs. In Section 5, a comprehensive numerical example will be presented to explain the properties and features of the suggested approach. Section 6 concludes the paper.

\section{Preliminaries and Technical Background}

Let us assume that there exist $n$ DMUs in the analysis. Let $X_{j}=\left(x_{1 j}, x_{2 j}, \ldots, x_{m j}\right) \in \mathfrak{R}_{+}^{m}$ and $Y_{j}=\left(y_{1 j}, y_{2 j}, \ldots, y_{s j}\right) \in \mathfrak{R}_{+}^{s}$ be non-zero vectors which quantify the level of inputs and outputs of $\operatorname{DMU}_{j}(j=1, \ldots, n)$. On this basis, the technology under which DMUs operate can be represented by a PPS or technology set of feasible input-output combinations as follows:

$$
P P S=\left\{(X, Y) \in \mathfrak{R}_{+}^{m} \times \mathfrak{R}_{+}^{s} \mid X \text { can produce } Y\right\}
$$

In terms of properties to be satisfied in a particular application, the PPS in (1) can precisely be characterized by applying a few mathematical axioms such as non-emptiness, free disposability, ray unboundedness (or CRS), convexity and minimum extrapolation. While applying these all axioms results in the CCR model proposed by Charnes, Cooper, and Rhodes (1978), removing 
the "ray unboundedness" assumption will change the CCR model to the BCC model of Banker, Charnes, and Cooper (1984).

Following Podinovski (2004a), an "absolute trade-off" represented by $\left(p_{k}, q_{k}\right)=\left(p_{1 k}, \ldots, p_{m k}, q_{1 k}, \ldots, q_{s k}\right), k=1, \ldots, K$, can also be added to the CCR model by means of an axiom as follows:

- Feasibility of absolute trade-offs: If $(X, Y) \in P P S$, then $(X, Y)+\pi_{k}\left(p_{k}, q_{k}\right) \in P P S$ such that $(X, Y)+\pi_{k}\left(p_{k}, q_{k}\right) \geq 0, k=1, \ldots, K$ for all $\pi_{k} \geq 0$.

where the resulting DEA model (e.g. the input-oriented one) to measure the efficiency score of a $\mathrm{DMU}_{\mathrm{o}}$ under evaluation can also be given as:

$$
\min \left\{\begin{array}{ll}
\theta_{o} \mid \begin{array}{ll}
\sum_{j=1}^{n} \lambda_{j} x_{i j}+\sum_{k=1}^{K} \pi_{k} p_{i k} \leq \theta_{o} x_{i o}, & i=1, \ldots, m, \\
\sum_{j=1}^{n} \lambda_{j} y_{r j}+\sum_{k=1}^{K} \pi_{k} q_{r k} \geq y_{r o}, & r=1, \ldots, s, \\
\lambda_{j}, \pi_{k} \geq 0, & j=1, \ldots, n, \quad k=1, \ldots, K, \\
\theta_{o} \text { free in sign }
\end{array}
\end{array}\right\}
$$

In order to show the effect of applying a trade-off in an absolute form, suppose a relative efficiency measurement system with a number of university departments as DMUs with a single input (e.g. number of employees) and two outputs (e.g. number of undergraduate students and number of master students, respectively). Taking into account the above axiom, a trade-off with a form of, e.g., $\left(p, q_{1}, q_{2}\right)=(0,-2,1)$ in this system means that a reduction in the number of undergraduate students with 2 units $\left(q_{1}=-2\right)$ should allow the DMUs to enlarge the number of master students with a single unit $\left(q_{2}=1\right)$ without requiring any additional employees $(p=0)$. As can be clearly observed in this approach, the volume of the inputs and outputs of the DMUs are not involved in the determination of the trade-offs. This can be seen by many DMUs as counterintuitive and a source of inequity regarding the way their efficiency is measured. 
As can be seen (and also shown by Alirezaee \& Boloori, 2012), the model in (2) is not units invariant either. This means that - unlike in the standard CCR and BBC DEA models efficiency scores determined by (2) depend on the units in which vectors of trade-offs are defined. Another remark given is that - in an optimal solution - model (2) may produce $\lambda_{j}^{*}=0$ for all $j$, and $\pi_{k}^{*} \neq 0$ for some $k$. In such situations, the DMU under evaluation is not really compared to other observed DMUs (or even any scale of a DMU or a convex combination of a few DMUs) but to a virtual DMU formed by a unit with "zero input-output quantities" and some positive trade-offs. This may also be considered as problematic in real applications where targetsetting is the fundamental objective of the relative efficiency measurement system (for more details, see Alirezaee \& Boloori, 2012; more details about target setting in DEA can also be found in, e.g. Lotfi et al. 2013; Hatami-Marbini et al. 2015b).

Against this background, Alirezaee and Boloori (2012) defined their "proportional" trade-offs, represented by $P T O_{k j}=\left(p_{k}, q_{k}\right) \otimes\left(X_{j}, Y_{j}\right)=\left(p_{1 k} x_{1 j}, \mathrm{~K}, p_{i k} x_{i j}, \mathrm{~K}, p_{m k} x_{m j}, q_{1 k} y_{1 j}, \mathrm{~K}, q_{r k} y_{r j}, \mathrm{~K}, q_{s k} y_{s j}\right)$, on the basis of the following axioms:

I. Feasibility of proportional trade-offs: If $\left(X_{j}, Y_{j}\right)$ is an observed DMU, then $\left(X_{j}, Y_{j}\right)+\pi_{k j} \cdot P T O_{k j} \in P P S$ such that $\left(X_{j}, Y_{j}\right)+\pi_{k j} \cdot P T O_{k j} \geq 0, k=1, \ldots, K, 0 \leq \pi_{k j} \leq a_{k}$, (i.e. $\pi_{k j}$ can also be defined unrestricted from above by $\left.a_{k}=+\infty\right)$

II. Aggregation of proportional trade-offs: If $\left(X_{j}, Y_{j}\right)$ is an observed DMU, then all trade-offs are collectively applied on it such that $\left(X_{j}, Y_{j}\right)+\sum_{k=1}^{K} \pi_{k j} \cdot P T O_{k j} \in P P S, 0 \leq \pi_{k j} \leq a_{k}$.

In order to show the effect of applying a trade-off in a proportional form, consider again the above outlined example of the relative efficiency system with the feasibility axiom (axiom I). A proportional trade-off with a form of, e.g., $\left(p, q_{1}, q_{2}\right)=(0,-2,1)$ in this example means that taking into account the volume of the unit under evaluation - a reduction of $2 \%\left(q_{1}=-2\right)$ in the number of undergraduate students allows for $1 \%\left(q_{2}=1\right)$ enlargement of the number of master students without requiring any additional employees $(p=0)$. Moreover, - similar to the approach of absolute trade-offs - the effect of a proportional trade-off is also allowed to be scaled. This 
will expand the PPS accordingly. For example, considering the trade-off of $\left(p, q_{1}, q_{2}\right)=(0,-2,1)$, any non-negative proportional scale of this trade-off - i.e. $\pi\left(p, q_{1}, q_{2}\right)=(0,-2 \pi, \pi)-$ will also be applied on the DMUs in the system. More precisely, if the units in this example are shown by $\left(x_{j}, y_{1 j}, y_{2 j}\right)$, all the feasible points on the line $\left(x_{j}, y_{1 j}, y_{2 j}\right)+\left(0,-2 \pi y_{1 j}, \pi y_{2 j}\right)$ will be in the PPS. On the one hand, this shows how the definition of a particular trade-off is adapted with respect to the volume of the input and outputs. ${ }^{1}$ On the other hand, it highlights the fact that a trade-off, e.g., of the form of $\left(p, q_{1}, q_{2}\right)=(0,-2,1)$, is scaled up or down by which it can appropriately be applied on a particular DMU with respect to its size. Hence, although the trade-offs can be defined basically between $0 \%$ and $100 \%$ (or 0 and 1), but a value of $\pi$ will automatically adjusts the defined percentages so that it can be imposed appropriately on a particular unit in the system according to its volume.

We also note that - unlike in the absolute trade-offs - within this approach, proportional tradeoffs are applied first on the "observed DMUs" (see axioms I and II). Then the PPS is expanded by employing further the other pre-selected axioms such as free disposability and convexity. With this, the analyst can ensure that the expanded PPS will not include areas which are only the results of imposing trade-offs on some "virtual units". As a direct consequence, the effect of trade-offs may also be individualized or restricted for the DMUs with an iteration variable $\pi_{k j}$, which can be set to be in a desired range as $\pi_{k j} \in D_{k}=\left[0, a_{k}\right] \subseteq \mathfrak{R}_{+}$. In this approach, different returns to scale can also be formed by an aggregation of appropriate proportional trade-offs. For example, one can apply NDRS (non-decreasing retunes to scale) with a trade-off vector $\left(p_{1 j}, q_{1 j}\right)=(1,1)$ with iteration domain $\pi_{1 j} \in[0, \infty)$. Similarly a trade-off vector $\left(p_{2 j}, q_{2 j}\right)=(-1,-1)$ with iteration domain $\pi_{2 j} \in[0,1]$ forms NIRS (non-increasing retunes to scale). Applying these two trade-offs jointly by means of the aggregation axiom (axiom II) produces CRS. Although these two trade-offs are applied only on original DMUs, but

\footnotetext{
1 This can be compared to the case that a similar trade-off is defined in an absolute form. Under the same circumstances, in the approach of absolute trade-offs, all feasible points on the line $\left(x_{j}, y_{1 j}, y_{2 j}\right)+(0,-2 \pi, \pi)$ will be included in the PPS. On this basis, the trade-off is applied on all DMUs by the same vector $(0,-2 \pi, \pi)$ which does not take into account the size of the DMUs in order to expand the PPS.
} 
considering also the effect of the other axioms such as convexity and free disposability, the whole PPS will satisfy the property of CRS globally (see Alirezaee and Boloori, 2012). Accordingly, there is no need to have the axiom "ray unboundedness" as a separate axiom.

The DEA model (e.g. the input-oriented one), which is founded on the basis of the axioms nonemptiness, free disposability, convexity, minimum extrapolation (hereafter we refer to the collection of these axioms as "basic axioms") together with the above additional feasibility and aggregation axioms, can be formulated as:

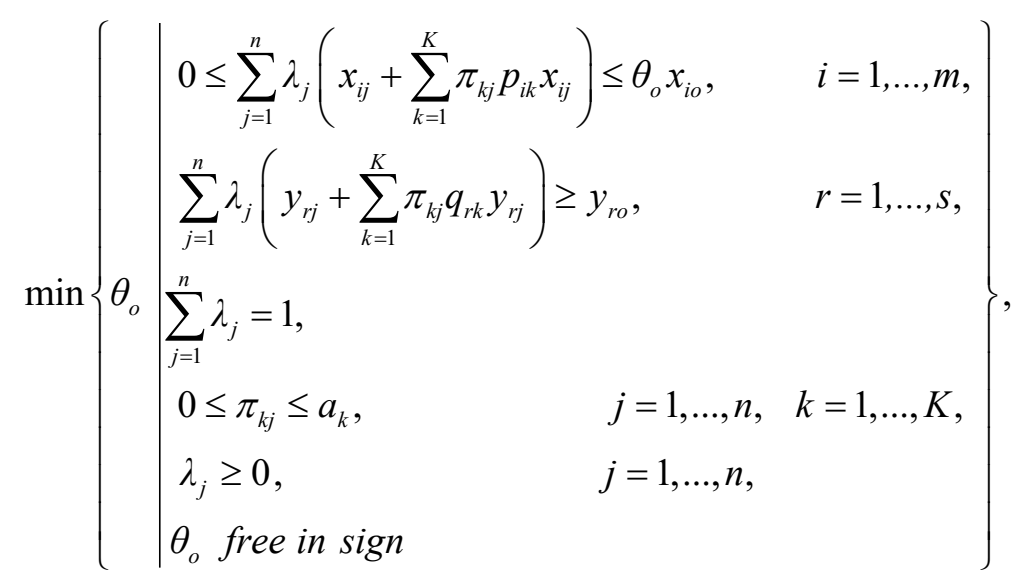

where model (3) can be transformed to the following linear model by substituting $\lambda_{j} \pi_{k j}$ with a single variable so that $\omega_{k j}=\lambda_{j} \pi_{k j}$ : 


$$
\min \left\{\begin{array}{l|l}
0 \leq \sum_{j=1}^{n} \lambda_{j} x_{i j}+\sum_{j=1}^{n} \sum_{k=1}^{K} \omega_{k j} p_{i k} x_{i j} \leq \theta_{o} x_{i o}, & i=1, \ldots, m, \\
\theta_{o}^{n} \lambda_{j} y_{r j}+\sum_{j=1}^{n} \sum_{k=1}^{K} \omega_{k j} q_{r k} y_{r j} \geq y_{r o}, & r=1, \ldots, s, \\
\sum_{j=1}^{n} \lambda_{j}=1, & j=1, \ldots, n, k=1, \ldots, K, \\
0 \leq \omega_{k j} \leq a_{k} \lambda_{j}, & j=1, \ldots, n, \\
\lambda_{j} \geq 0, & \\
\theta_{o} \text { free in sign } &
\end{array}\right\} .
$$

In this model, efficiency scores are computed while the trade-offs are determined proportionate to the volume of the inputs and outputs of DMUs. Hence, this model is units invariant in the sense that efficiency scores determined by (4) do not depend on the units in which inputs and outputs are defined. It should also be noted that if in an optimal solution of (4), $\lambda_{j}^{*}=0$ for a specific $j$, hence automatically $\omega_{k j}{ }^{*}=0$ for all $k$. This implies that if a real DMU is not considered as a reference in the results (i.e. $\lambda_{j}^{*}=0$ ), the trade-offs will not be applied on this DMU either (i.e. $\omega_{k j}{ }^{*}=0$ ), avoiding the case that a DMU is compared to a virtual DMU formed by a unit with "zero input-output quantities" and some positive trade-offs. Other advantages of this approach can be found in Alirezaee and Boloori (2012).

We complete this section by a guideline on the use of proportional trade-offs. Proportional tradeoffs - as an enhanced form of the absolute trade-offs - can be used to incorporate prior views or information regarding the assessment of DMUs into relative efficiency measurement systems by DEA. Different situations can be recognized in which such trade-offs can also be applied by the analyst in order to ensure that the results are not counter-intuitive. Allen et al. (1997) give an interesting classification of weight restrictions in order to incorporate value judgments into DEA analysis. As absolute trade-offs are seen as the dual form of weight restrictions, we believe that this classification by Allen et al. (1997) can still be used as a general guideline for situations where proportional trade-offs need to be applied. Therefore, in the following, we adapt their work and propose a guideline on the application of proportional trade-offs. According to this 
guideline, one can find out in which situations and how the proportional trade-offs could also be applied.

\section{- Value of individual inputs and outputs:}

In some situations, managers may have prior views on the relative importance of the inputs and outputs in the analysis. These judgements can be extracted from the manager's perspectives and incorporated into the analysis by proportional trade-offs. The translation of judgments into tradeoffs could be done in a similar way which has been followed by, e.g., Thanassoulis et al. (1978) or Dyson and Thanassoulis (1988) for the case of weight restrictions. It should also be noted that some researchers have recently proposed analytical hierarchy approach (AHP) to be used for an accurate translation of value judgments into weight restrictions (Sinuany-Stern, Mehrez, \& Hadad, 2000; Premachandra, 2001; Wang, Parkan, \& Lu, 2008). An appropriate adaption of such approaches may also be used for the case of proportional trade-offs.

\section{- Dependency between inputs and/or outputs:}

In some applications, some input/output factors may have direct rational dependence. Accordingly, managers may wish to include these relations in advance in the analysis. For example, Podinovski and Wan Hossein (2015) use absolute trade-offs to incorporate an additional judgement about the relation between the number of academic staff and the number of academic research works into the analysis. Such a view can be translated similarly by proportional trade-offs. Some other data mining methods have also been proposed recently in order to recognize patterns behind the relations between inputs and/or outputs (see, e.g., Nadimi and Jolai, 2008: Mecit and Alp 2013). Bringing these methods to the applications of proportional trade-offs seems to be also applicable

\section{- Prior ideas about the performance results:}

In some scenarios, decision makers may have some priori ideas about the performance of units under assessment. For example, they may have a classification of "good" and "poor" performers in the analysis. Such a judgment can be added to the analysis by means of proportional trade-offs 
in a similar manner as it has already been done for the case of weight restrictions by means of the idea of cone ratio (see, e.g., Charnes et al. 1990). Such an approach could still be used for the case of applying proportional trade-offs.

\section{- Marginal rate of substitution:}

Marginal rate of substitution is an economic concept which provides the analyst with information about the substitution between the inputs and outputs in the analysis. According to Charnes et al. (1978), the ratio of the input to output weights by DEA can also give an estimation of such rates between the inputs and outputs. It has already been shown how additional value judgments can be used in the form of weight restriction to prevent this ratio of being zero or restricting it to vary only in a desired range (see e.g. Bessent et al. 1988 and Olesen and Petersen, 1991). As proportional trade-offs can limit such substitutions, an appropriate definition of trade-offs can also be used in the analysis in a similar way.

\section{- Discriminatory power of the models}

In some DEA applications, the results are not enough discriminated in such a way that there are a large portion of efficient units. This makes the analysis impractical as the efficient units cannot receive any suggestions for further improvement. One way to improve the discriminatory power of a DEA model is imposing additional value judgements in the model. For example, Podinovski (2007) provides some practical suggestions of how absolute trade-offs can be incorporated into a model for such purposes. As the approach with proportional trade-offs modifies the definition of the absolute trade-offs, such suggestions could also be used in the related applications.

\section{Consistency in Proportional Trade-offs}

As outlined in the introduction, the incorporation of trade-offs might lead to a set of inconsistent restrictions imposed to the corresponding DEA models. This will then generate serious problems such as infinity or even negative efficiency scores in the results. In the following, we investigate situations in which imposing an "individual proportional trade-off" can result in an unexpected expansion of the PPS. Furthermore, mathematical programming models are also formulated to 
test if "all proportional trade-offs" are consistent so that they can be applied simultaneously or not. Note here that, in the following, we may refer simply to "trade-offs" as "proportional tradeoffs".

\subsection{The Case of Infeasible Expansion}

In order to demonstrate the first potential problem of imposing inconsistent proportional tradeoffs, let us consider the simple case depicted in Fig. 1 of four DMUs with a single input (all DMUs have the same level of input) and two outputs. The basic PPS before applying the feasibility and aggregation axioms, which is bounded by ABCD, is shown in Fig. 1(a).

Fig. 1. The case of infeasible expansion

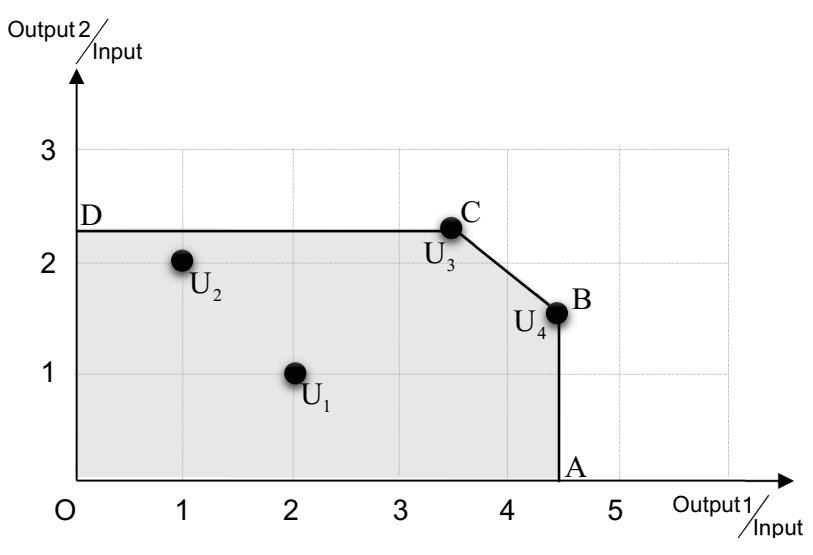

(a)

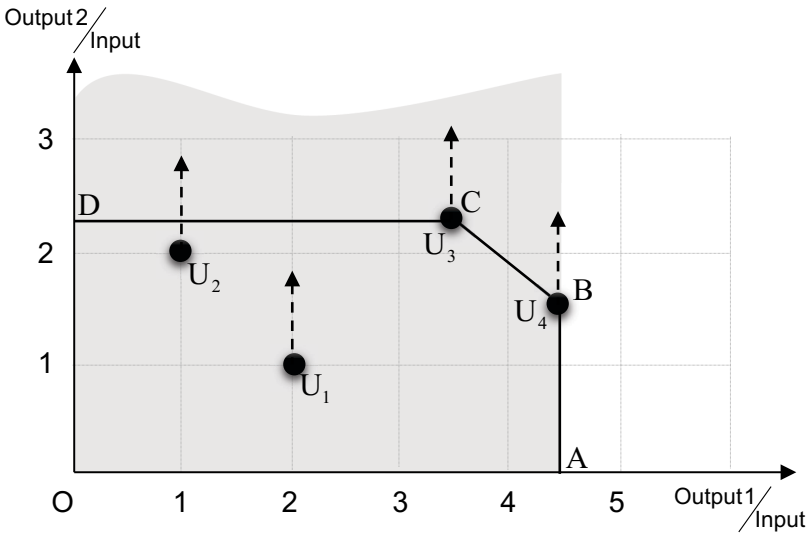

(b)

Let us assume that a proportional trade-off with a form of $\left(p, q_{1}, q_{2}\right)=(0,0,2)$ is applied. This means that the second output of each DMU can be enlarged with $2 \%\left(q_{2}=2\right)$ of the corresponding volume of this output without any reduction of the first output $\left(q_{1}=0\right)$. If we also apply the other basic axioms and expand the PPS accordingly, we can clearly see that this results in an infinite enlargement of the PPS in a direction of output. This simple example clarifies that an enlargement of any output without a reduction of at least another output or an increase of at least an input will result in an "infeasible expansion" of the PPS. Taking into account the input side, we can similarly deduce that any reduction of an input must also result in a reduction of at least an output or an enlargement of at least another input in the analysis. Otherwise, a case of 
infeasible expansion happens and the corresponding trade-off is considered as invalid since there is no free lunch in reality.

Taking a closer look at the above example reveals that the case of infeasible expansion occurs in a PPS if and only if a trade-off can be written as a non-negative linear combination of the two vectors $-e_{i}^{x}=\left(0, \ldots, 0,{\underset{i^{t h}}{1}}^{1}, 0, \ldots, 0\right)$ and $e_{r}^{y}=\left(0, \ldots, 0,{\underset{r}{r^{t h}}}_{\{}^{1}, 0, \ldots, 0\right), i=1, \ldots, m$ and $r=1, \ldots, s$. For example, the above trade-offs, $\left(p, q_{1}, q_{2}\right)=(0,0,2)$ and $\left(p, q_{1}, q_{2}\right)=(0,2,2)$, can be written as $0 e_{1}^{x}+0 e_{1}^{y}+2 e_{2}^{y}$ and $0 e_{1}^{x}+2 e_{1}^{y}+2 e_{2}^{y}$, respectively. This simple test provides us with a tool to identify if a particular trade-off expands the PPS in an infeasible manner. However, such an unexpected expansion can also happen from applying all trade-offs simultaneously. The reason is that imposing more than one trade-off, the convexity and/or aggregation axiom may also expand the PPS in an infeasible way. In order to check if all trade-offs together may lead to an acceptable enlargement of the PPS, we extend our test as follows:

Definition 1: Let $\Omega=\left\{\left(p_{k}, q_{k}\right) \mid k=1, \ldots, K\right\}$ be the full set of vectors of proportional trade-offs defined in the analysis. According to the feasibility axiom, the set $\Delta(\Omega)=\left\{\sum_{k=1}^{K} \pi_{k}\left(p_{k}, q_{k}\right) \mid 0 \leq \pi_{k} \leq a_{k}, \forall k\right\}$ represents a cone generated by all trade-offs. On this basis, imposing all trade-offs collectively leads to an infeasible expansion if and only if the cone generated by $e_{r}^{y}=\left(0, \ldots, 0, \underset{r_{-} t h}{1}, 0, \ldots, 0\right)$ and $-e_{i}^{x}=\left(0, \ldots, 0, \underset{i_{-} t h}{1}, 0, \ldots, 0\right)$ has an intersection with $\Delta(\Omega)$.

Now, the following theorem provides a mathematical tool to check the result of the above definition.

Theorem 1: With respect to definition 1, imposing all trade-offs simultaneously leads to an infeasible expansion of the PPS if and only if the optimal value of model (5) becomes nonzero. 


$$
\rho_{1}=\max \left\{\begin{array}{l|ll}
\sum_{i=1}^{m} w_{i}^{x}+\sum_{r=1}^{s} w_{r}^{y} & \begin{array}{lll}
\sum_{k=1}^{K} \pi_{k} p_{i k}=-w_{i}^{x}, & i=1, \ldots, m, & \\
\sum_{k=1}^{K} \pi_{k} q_{r k}=w_{r}^{y}, & r=1, \ldots, s, \\
0 \leq \pi_{k} \leq a_{k}, & k=1, \ldots, K, \\
w_{i}^{x}, w_{r}^{y} \geq 0 & i=1, \ldots, m ; \quad r=1, \ldots, s
\end{array}
\end{array}\right\} .
$$

Proof. We note here that $w_{i}^{x}=w_{r}^{y}=\pi_{k}=0$ (for all $i, r$ and $k$ ) represents a feasible solution. Hence, this model is always feasible. According to definition 1, in the case that the imposed trade-offs are consistent, $\Delta(\Omega)$ has no intersection with the cone generated by $e_{r}^{y}$ and $-e_{i}^{x}$. This means that there has to be no non-negative linear combination of ${\stackrel{\mathrm{r}}{\boldsymbol{e}_{r}}}_{r}$ and $-\mathrm{r}_{i}^{x}$ in the linear combination of the trade-offs. In other words, if a non-negative linear combination of the tradeoffs satisfies statement (6), it implies that $w_{i}^{x}=w_{r}^{y}=0$ for all $i$ and $r$ :

$$
\sum_{k=1}^{K} \pi_{k}\left(p_{k}, q_{k}\right)=\sum_{i=1}^{m} w_{i}^{x}\left(-e_{i}^{x}\right)+\sum_{r=1}^{s} w_{r}^{y}\left(e_{r}^{r_{r}}\right)
$$

The right hand side of the term given in (6) clarifies the existence of the constraints in model (5). To check the inverse of the theorem, let us assume that in the optimal solution of (5) $\sum_{i=1}^{m} w_{i}^{* x}+\sum_{r=1}^{s} w_{r}^{* y}=0$. Since the problem is maximization, there could have not been positive solutions for $w_{i}^{x}$ and $w_{r}^{y}$ (otherwise the objective function and accordingly the optimal objective function value would have been greater than zero). With respect to (6), there is no positive linear combination of $\stackrel{\mathbf{r}}{e}_{r}^{y}$ and $-\stackrel{\boldsymbol{r}}{e}_{i}^{x}$ in the linear combination of the trade-offs. This implies that the sets of all trade-offs are consistent.

\subsection{The Case of Free Production}

In order to demonstrate the second potential problem of imposing inconsistent proportional trade-offs, consider first the following graphical example in which there are four DMUs with a single input and a single output. The basic PPS (before applying the feasibility and aggregation axioms), which is bounded by ABCDE, is shown in Fig. 2(a). 
Fig. 2. The case of free production

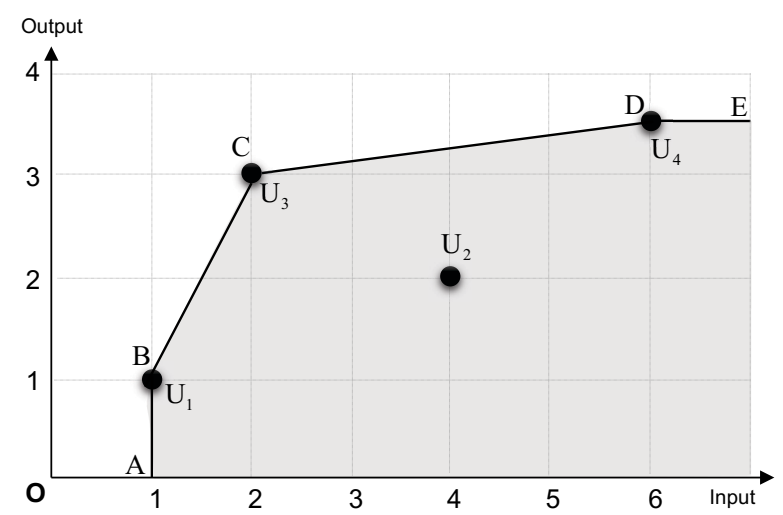

(a)

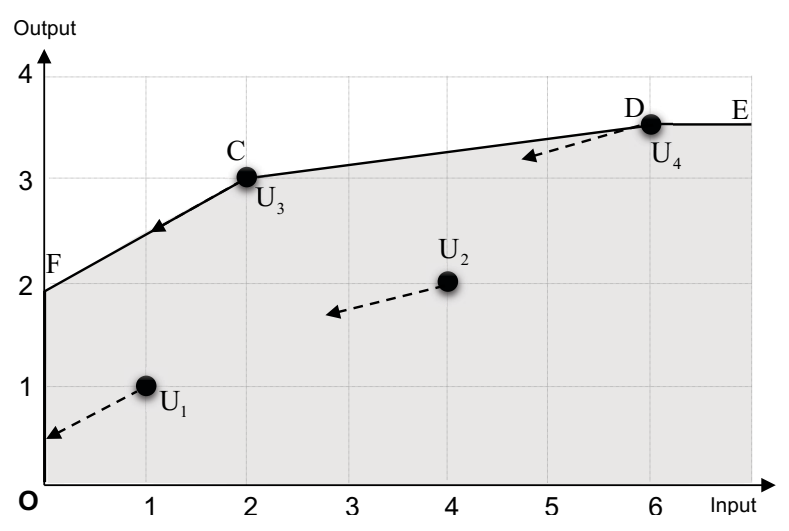

(b)

Let us suppose that a proportional trade-off with a form of $(p, q)=(-2,-1)$ is applied. This means that the output of each DMU will decrease with $1 \%(q=-1)$ of the volume of the output if the input is reduced by $2 \%(p=-2)$ of the volume of the input. If we also apply the other basic axioms, the expanded PPS, which is shown in Fig.2 (b), is bounded by OFCDE. From this figure, we can see that the PPS now includes the line OF on the output axis, meaning that there is a case of "free production" in the PPS, i.e. production with zero input. The reason is that although both the input and output are reduced according to the definition of this trade-off, but the change in the input is faster than that in the output. Therefore, virtual units are added to the PPS (i.e. those on the line OF) which have the ability to produce the output with nothing! This obviously represents an invalid relation between the input and output in the PPS, which should be avoided. This case of "free production" in a PPS resulting from imposing either a single tradeoff (like in this example) or multiple trade-offs can mathematically be identified as follows:

Definition 2: Let $\Omega=\left\{\left(p_{k}, q_{k}\right) \mid k=1, \ldots, K\right\}$ be the full set of vectors of proportional trade-offs imposed to the PPS. Free production exists in the expanded PPS if and only if $(0, \hat{Y})$ belongs to this PPS such that $\hat{Y} \geq 0, \hat{Y} \neq 0$.

The following theorem also provides a mathematical tool to check the result of the above definition. 
Theorem 2: With respect to definition 2, imposing all trade-offs simultaneously leads to the case of free production in the PPS if and only if the optimal value of the following model becomes nonzero.

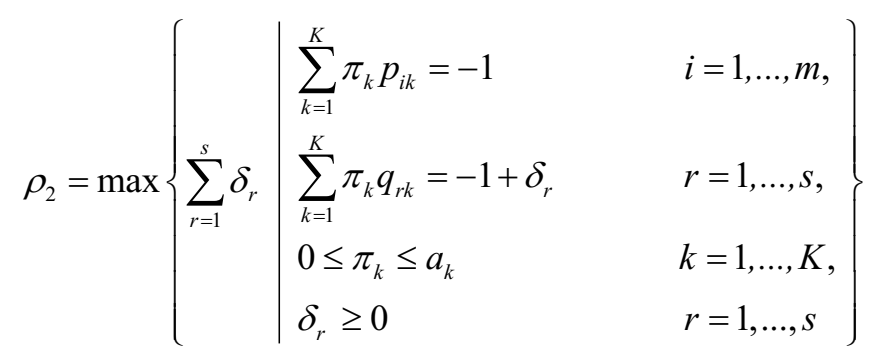

Proof. Consider $\left(X_{j}, Y_{j}\right)$ as an observed unit. According to definition 2, the effect of the imposed trade-offs on this DMU produces the case of free production if and only if there exist some $\hat{Y}=\left(\hat{y}_{r}\right) \geq 0, \hat{Y} \neq 0$ and also $\pi_{k j}$ in which:

$$
\begin{cases}x_{i j}+\sum_{k=1}^{K} \pi_{k j} p_{i k} x_{i j}=0, & i=1, \ldots, m, \\ y_{r j}+\sum_{k=1}^{K} \pi_{k j} q_{i k} y_{r j}=\hat{y}_{r}, & r=1, \ldots, s, \\ 0 \leq \pi_{k j} \leq a_{k}, & k=1, \ldots, K .\end{cases}
$$

In (8), we can replace $\hat{y}_{r}$ by $\alpha_{r} y_{r j}$ such that $\alpha_{r} \geq 0, \quad r=1, \ldots, s$. Since $\hat{Y} \neq 0$, it implies that $\alpha=\left(\alpha_{1}, \ldots, \alpha_{s}\right) \neq 0$. By means of this substitution of variables, $x_{i j}$ and $y_{r j}$ can be omitted such that the equations in (8) do not depend to the data of $\mathrm{DMU}_{j}$. Therefore, variable $\pi_{k j}$ can also be replaced by $\pi_{k}$. Now we can rewrite (8) in the form of (9) and conclude that the case of free production occurs if and only if: 


$$
\begin{cases}\sum_{k=1}^{K} \pi_{k} p_{i k}=-1, & i=1, \ldots, m, \\ \sum_{k=1}^{K} \pi_{k} q_{r k}=-1+\alpha_{r}, & r=1, \ldots, s, \\ 0 \leq \pi_{k} \leq a_{k}, & k=1, \ldots, K .\end{cases}
$$

As can be seen now, the constraints of model (7) are the same as in (9). Therefore, the case of free production occurs if and only if the model in (7) have a solution with a strictly positive objective function $\sum_{r=1}^{s} \alpha_{r}$.

\section{The Overall PPS of Consistent Trade-offs}

Taking into account the previous definitions and the corresponding theorems we can check if a particular trade-off can be imposed successfully or not, i.e. an individual test is carried out. Furthermore, we can also investigate whether the set of "all trade-offs" together expand the PPS in an acceptable way, i.e. a global test is done. According to the discussions in the previous section, the mechanism of this global test can be summarized as follows:

Remark 1: A set of Trade-offs $\Omega=\left\{\left(p_{k}, q_{k}\right) \mid k=1, \ldots, K\right\}$ is consistent if and only if both the following conditions are satisfied:

- in model (5), $\rho_{1}=0$.

- in model (7), either $\rho_{2}=0$ or the model is infeasible.

Now let us assume, in a specific context, that the individual test does not identify any problem but the global test does. This means that applying a single trade-off at a time does not result in a problem. However, mathematical issues (such as infeasible expansion or free production) occur when the trade-offs are applied all together. Assuming that each trade-off has been defined carefully and has its own particular meaning in the context, what can best be said is straightforward: 
There exist a few trade-offs which cannot be aggregated in "a conventional way" to expand the PPS. In other words, the existing framework by which the trade-offs are combined mathematically to form the PPS cause a problem rather than the definition of the trade-offs. Against this background, in the following section, we revisit the conventional approach of combining trade-offs. We identify shortcomings in this approach and use it as a starting point to develop a new approach of aggregating trade-offs, which avoids the problems outlined in the previous sections.

\subsection{Motivation}

Consider a relative efficiency system with a single input and two outputs. Let us simplify the case and assume that there exist only two DMUs, which are denoted by $\mathrm{U}_{1}$ and $\mathrm{U}_{2}$. The basic PPS before applying the feasibility and aggregation axioms, shown in Fig. 2, is bounded by ABCD.

Fig. 3. Local $\mathrm{PPS}_{1}$ and $\mathrm{PPS}_{2}$

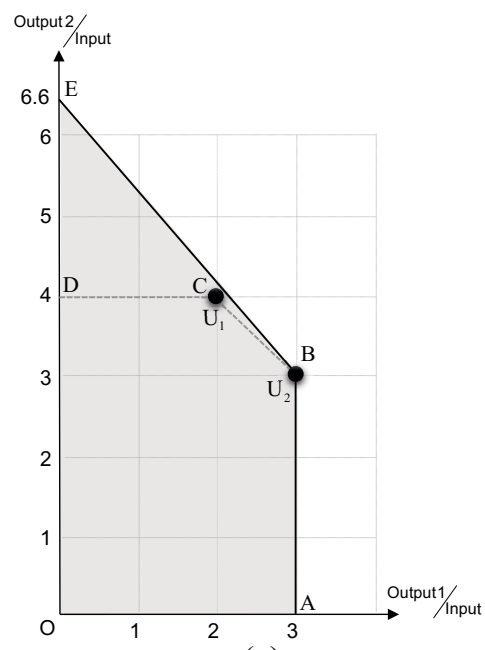

(a)

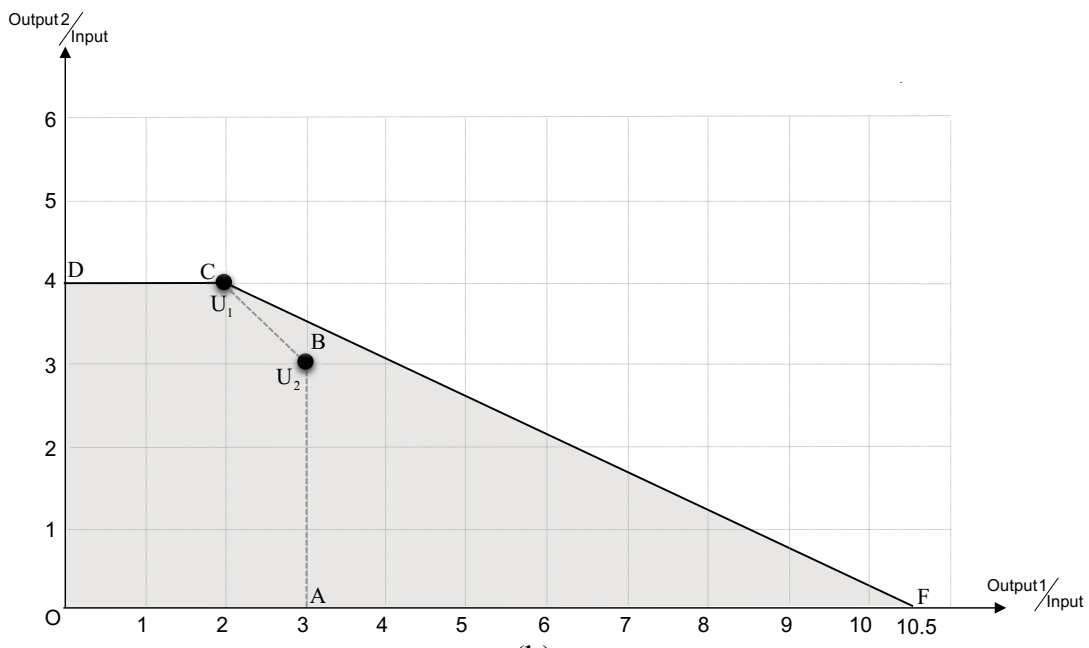

(b)

Let us suppose that a proportional trade-off is applied with a form of $T_{1}=\left(p, q_{1}, q_{2}\right)=(0,-0.3,0.2)$ and an iteration domain in $\mathfrak{R}^{+}$. This means that the second output of each DMU can be enlarged with $0.2 \%\left(q_{2}=0.2\right)$ of the volume of this output while the first output is reduced by $0.3 \%\left(q_{1}=-0.3\right)$ of its corresponding volume. Checking the trade-off with the proposed models in Section 3, we can recognize that it can mathematically be applied 
without any problem. This can also be observed from the graphical representation of the corresponding expanded PPS, which is depicted in Fig. 3(a). We refer to this expanded PPS, which is bounded by ABE, simply as PPS $_{1}$. Similarly let us impose another trade-off with a form

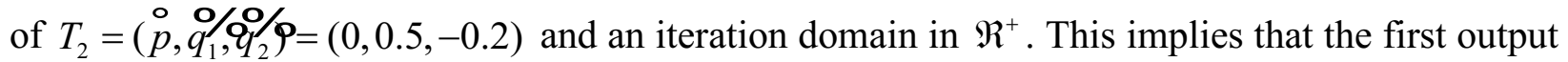
of each DMU can be enlarged with $0.5 \%\left(q_{1}=0.5\right)$ of the volume of this output while the second output is reduced by $0.2 \%\left(q_{2}=-0.2\right)$ of its corresponding volume. Having applied this tradeoff, the resulting expanded PPS (i.e. $\mathrm{PPS}_{2}$ bounded by FCD) has been presented in Fig. 3(b). Not only graphically, but also by means of the proposed models in the previous section, one can recognize that this trade-off can be imposed without any mathematical problem as discussed in Section 3.

Now, let us apply these two trade-offs jointly. The resulting PPS is depicted in Fig. 4. As can be seen (it could also be checked mathematically by means of the proposed models in Section 3), we end up with an unexpected infinite enlargement of the PPS.

Fig. 4. The expanded PPS formed by the conventional approach

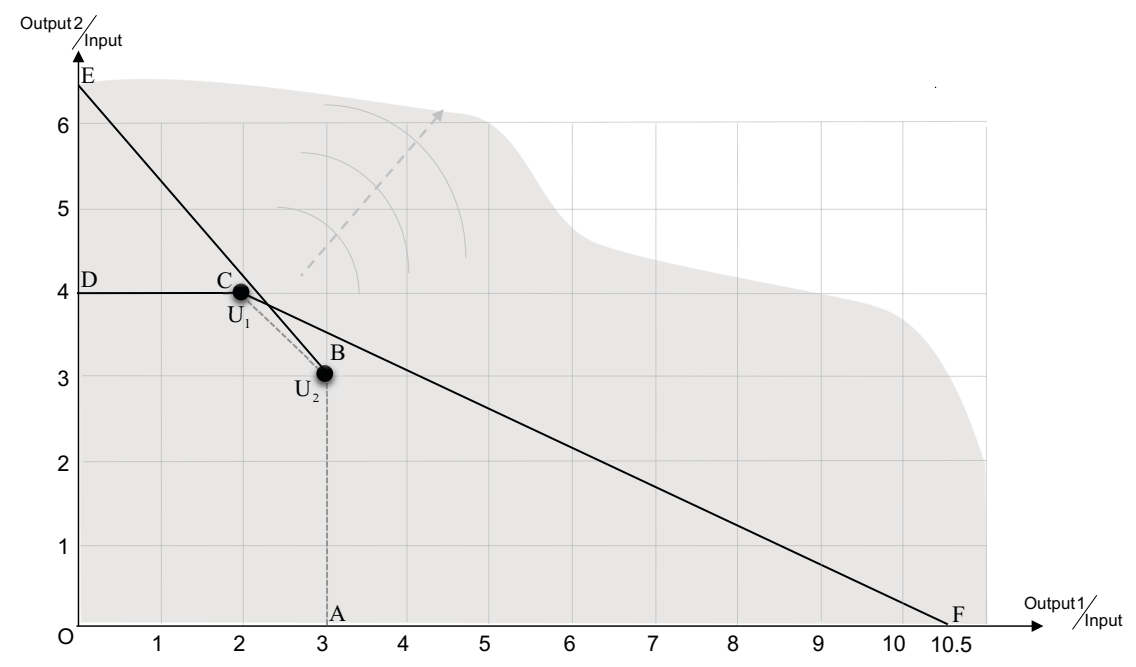

As these two trade-offs can successfully be applied separately, they are inconsistent only with respect to the way that the corresponding local $\mathrm{PPS}_{1}$ and $\mathrm{PPS}_{2}$ are aggregated. More precisely, not only the aggregation axiom, but also the convexity axiom adds areas to the PPS which expand it into infinity. As the reason for this phenomenon is only of a mathematical one - i.e. the way the aggregation and convexity axioms are employed -, we propose a new aggregation which 
does not necessitate any further assumptions to be made for the union of the local PPSs. This alternative union can be formed by applying the minimum extrapolation principle on the union of the $\mathrm{PPS}_{1}$ and $\mathrm{PPS}_{2}$ as $P P S=P P S_{1} \cup P P S_{2}$. The means that although the convexity and aggregation assumptions can be applied locally in each PPS, any combination (either by the aggregation axiom or by the convex combination) of observations in these two PPSs is not permitted to form the final PPS. This pure union of the PPSs - shall be called in the following as "overall PPS" - is shown in Fig. 5.

Fig. 5. The overall PPS formed by the proposed approach

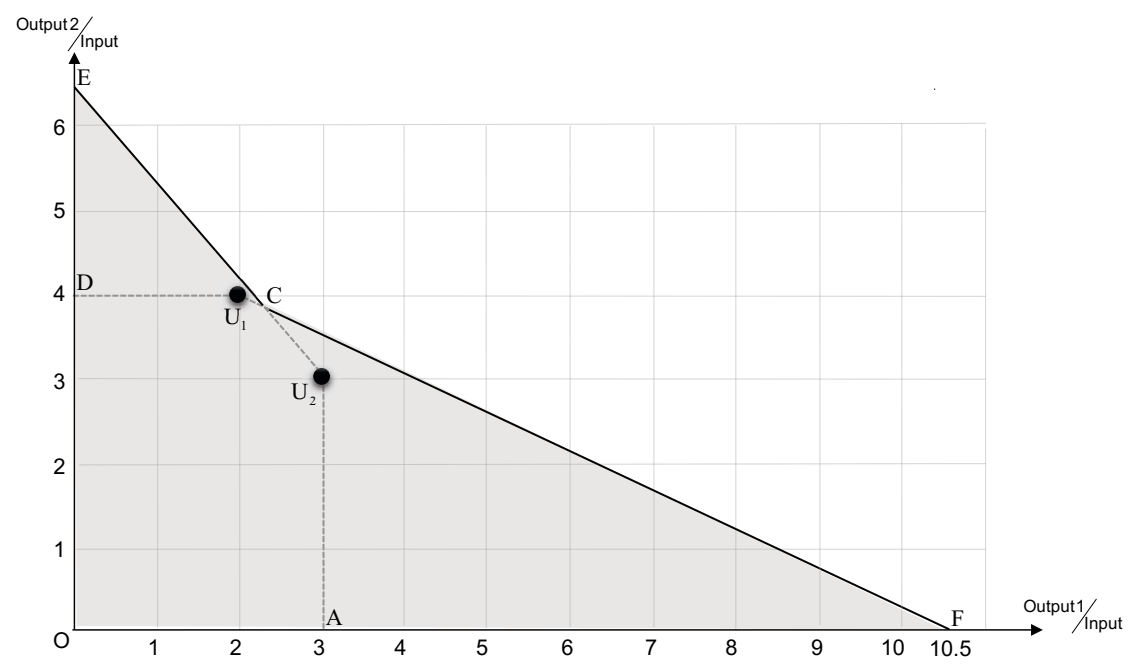

As can be seen in Fig 5., this PPS, which is now bounded by FCE, combines the local PPSs but only in such a way that the final expanded PPS excludes purely mathematical combinations of observations which neither been experienced nor can be producible in reality. Another interesting feature of the proposed method of aggregation is that individual characteristics of the local PPSs can be preserved and later traced in measuring efficiency. This is of particular importance in real applications where inefficient DMUs normally wish to learn from benchmark units. In such situations, the assigned benchmarks to inefficient DMUs come from a specific local PPS. This guarantees that - unlike in the conventional approach - the benchmarks are not generated by some pure mathematical combinations of a number of other points which belong to the PPS only because of the convexity and/or aggregation axioms applied on the trade-offs. 
In this example, there were only two trade-offs. However, in real world applications there is likely to be more than two trade-offs. In such cases, we need first to recognize which subsets of trade-offs can be collectively applied to enlarge the PPS. We shall refer to such subsets as "consistent subsets". As will be shown in the following section, having found all consistent subsets of trade-offs, we can use the maximal subsets of these trade-offs (i.e. those subsets with the maximum number of consistent trade-offs whose union is the original set of trade-offs) to define local PPSs to be aggregated to form the overall PPS.

\subsection{Determination of Maximal Sub-sets with Consistent Trade-offs}

In the following, we propose a tree-search procedure and its respective algorithm by which the trade-offs are grouped into a few subsets with the maximum number of "consistent trade-offs" which can be applied together. We refer to these subsets as "maximal consistent subsets". These subsets will be used later to define local PPSs, which form collectively the overall PPS (see Section 4.1) within our approach.

Let $\Omega=\left\{\left(p_{k}, q_{k}\right) \mid k=1, \ldots, K\right\}$ be the full set of trade-offs, which are expected to be imposed in the analysis. Let us assume that an individual test (i.e. trade-offs are checked one by one, not collectively) is carried out and the set $\Omega$ is refined in such way that no particular trade-off is further identified as invalid. Note that if a "particular trade-off" is invalid, its definition must be revisited by the decision maker at the early stage of the analysis. Accordingly, in the following, we assume that each subset with a single member can be applied without any mathematical difficulty as the individual test has already been carried out and the original set has been refined in advance. Suppose, on the other hand, that $\Omega$ contains a few inconsistent trade-offs which cannot collectively be applied in a conventional way. This phenomenon can be recognized by the proposed models in (5) and (7) (see also the global test given as Remark 1).

Disregarding the original set (i.e. the set with the cardinality $|\Omega|=K$ ), the empty set and also subsets with a single member only, there will be $2^{k}-k-2$ non-obvious subsets of $\Omega$, which may include inconsistent trade-offs. The generation of all maximal sets of consistent trade-offs can be represented by means of a tree-search procedure whose corresponding pseudocode is given in 
Algorithm \#1. In this algorithm, while $\Omega$ represents the set of all trade-offs given as its input, the output of the algorithm will be shown by $\Gamma=\left\{\Gamma_{1}, \ldots, \Gamma_{C}\right\}$, which collects the maximal sets of consistent trade-offs. Set $\Pi$ also collects all sets which are to be checked for consistency in each level of the tree search.

In the initialization step of the algorithm, the entire set of trade-offs is placed in the root of the tree (see line 1-2 on which $\Pi=\left\{\Pi_{1}\right\}=\{\Omega\}$ ). Therefore, at the beginning, set $\Omega$ is the only member of $\Pi$ which will be branched. Moreover, since no consistent set is identified yet, $\Gamma=\phi$ (see line 3). Parameters $B$ and $C$ as also represent the cardinalities of $\Pi$ and $\Gamma$ respectively.

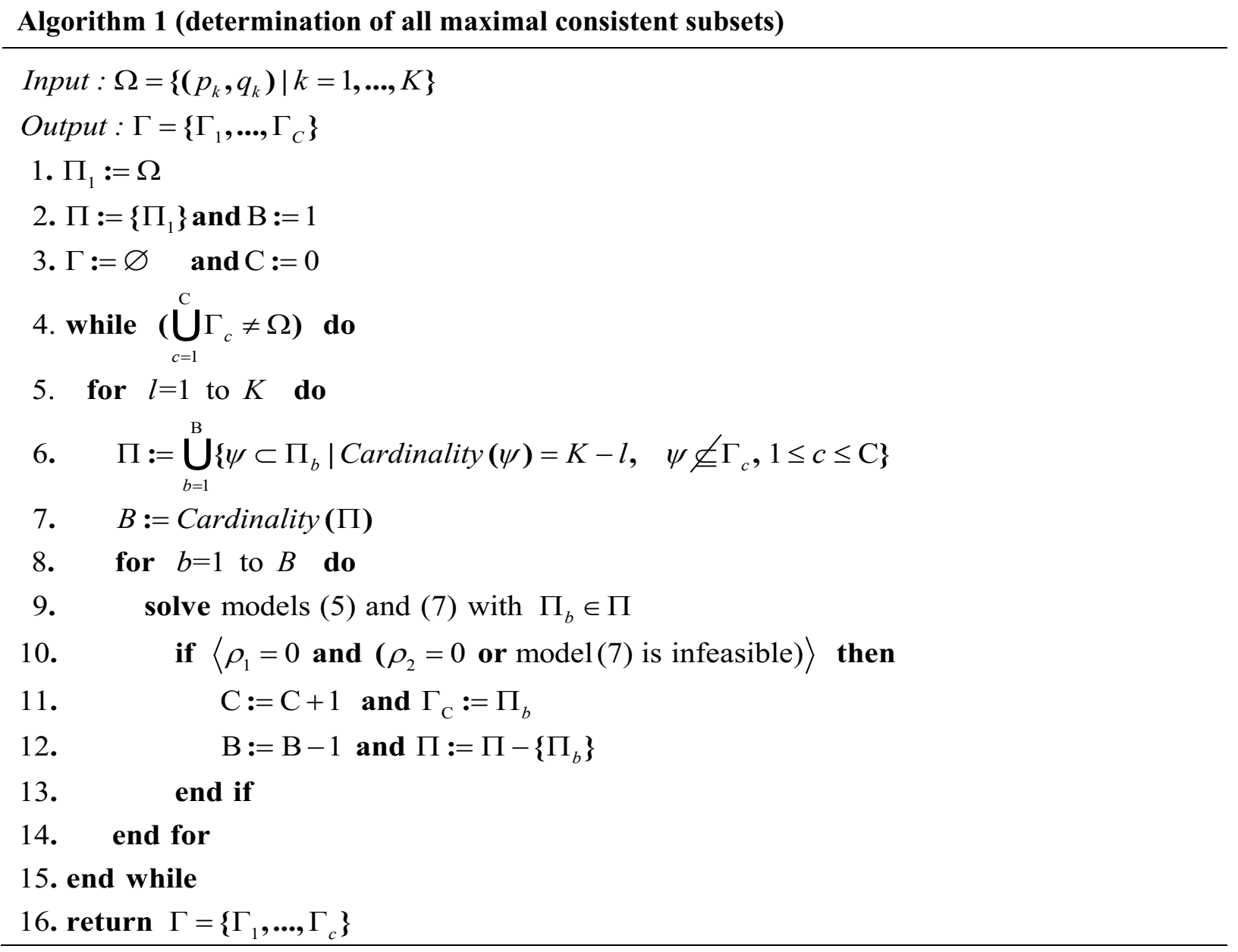


As can be seen on line 4 of the algorithm, it is checked whether all trade-offs exist in at least one of the already identified maximal consistent subsets (i.e. $\bigcup_{c=1}^{\mathrm{C}} \Gamma_{c} \neq \Omega$ ). If such situation occurs, all trade-offs have already been covered by a number consistent sets and further branching is not necessary. The reason is that any set in further branches would be a subset of one of the consistent sets in $\Gamma$. Hence, such a subset will not be maximal.

In line 6 of the algorithm we use a branching rule which shows how set $\Pi$ collects all sets which are to be checked for consistency. According to this rule, after branching in each level, each member $\Pi_{b}$ of $\Pi$ is checked for consistency by means of the proposed models in (5) and (7) (see line 9 of the pseudocode). With respect to this condition, two cases may happen:

1. If a member $\Pi_{b}$ of $\Pi$ is recognized to be consistent, its subsets are obviously consistent and $\Pi_{b}$ should not be branched (its branches are smaller consistent sets). Therefore, it is discarded from $\Pi$ and is transferred to the set $\Gamma$ as a consistent set (i.e. $C$ increases by 1 so that the last newly added member of $\Pi$ becomes $\Gamma_{\mathrm{C}}:=\Pi_{b}$; See also lines 10-12 in the algorithm).

2. If a member $\Pi_{b}$ of $\Pi$ is not consistent, it remains in $\Pi$ for probable further branching and consistency check in its subsets in the next step of the algorithm.

Suppose, in one step of the algorithm (where $l$ changes), that inconsistent sets in $\Pi$ (from the previous step) must be branched. Now, one may decide on how to branch at a minimum. Here we apply another branching rule which is used to avoid some redundant consistency checks. Let us assume that $\Pi_{b}$ as a member in $\Pi$ must be branched. Among its subsets (like $\psi$ ) with cardinality $\left|\Pi_{b}\right|-1$, those are considered for further branching that they are not included in any consistent subsets which have been recognized yet (i.e. $\psi \not \subset \Gamma_{c}$, see line 6 in pseudocode). This is because if it happens, $\psi$ would be a smaller consistent subset and its consistency check is redundant. This test also prevents the search from the entrance of non-maximal consistent sets into $\Gamma$ (we should search only for maximal consistent subsets). Consequently, only those 
$\psi \subset \Pi_{b}$ with cardinality $\left|\Pi_{b}\right|-1$ are considered as a new branch, in which they are not subsets of any $\Gamma_{c}$. Now the new $\Pi$ is refreshed so that it consists of the newly generated branches.

As it will be shown in the next section, these maximal consistent subsets will be used to define the local PPSs within our approach. The efficiency is then measured against the overall PPS, which is formed by the pure union of the local PPSs outlined already graphically in Section 4.1.

\subsection{Efficiency Measurement with the Overall PPS}

Let $\Omega=\left\{v_{k} \mid v_{k}=\left(p_{k}, q_{k}\right), k=1, \ldots, K\right\}$ be the entire set of proportional trade-offs. Having run the proposed algorithm given in the previous section, let us assume that we end up with $C$ maximal consistent subsets (i.e. $\Gamma_{\max }=\left\{\Gamma_{1}, \ldots, \Gamma_{c}, \ldots, \Gamma_{C}\right\}$. According to the graphical discussions in Section 4.1, the trade-offs in each $\Gamma_{c}$ can be applied collectively to form a corresponding local PPS shown by $P P S_{\Gamma_{c}}$. Similar to the graphical example given in Section 4.1, the overall PPS is then formed by the pure union of all local PPSs as:

$$
P P S=\bigcup_{c=1}^{C} P P S_{\Gamma_{c}}
$$

Although this representation is conceptually useful, a more precise model of the overall PPS can be derived by considering a number of mathematical axioms as follows:

1. (Non-emptiness). The observed $\left(X_{j}, Y_{j}\right) \in P P S, j=1, \ldots, n$.

2. (Free disposability). If $(X, Y) \in P P S, X^{\prime} \geq X, Y^{\prime} \leq Y$, then $\left(X^{\prime}, Y^{\prime}\right) \in P P S$.

3. (Local convexity). If $(X, Y)$ and $(X \%, Y / \rho \in P P S$, then $\lambda(X, Y)+(1-\lambda)(X \%, Y / \rho \in P P S$ for any $\lambda \in[0,1]$, provided that there exists $c(c=1, \ldots, C)$ such that both $(X, Y)$ and $\left(X / Y, Y / \varphi \in P P S_{\Gamma_{c}}\right.$.

4. (Feasibility). If $\left(X_{j}, Y_{j}\right)$ is an observed DMU, then $\left(X_{j}, Y_{j}\right)+\pi_{k j}$. PTO $O_{k j} \in P P S$ such that $\left(X_{j}, Y_{j}\right)+\pi_{k j} . P T O_{k j} \geq 0,0 \leq \pi_{k j} \leq a_{k}$. 
5. (Local aggregation). $(X, Y)=\left(X_{j}, Y_{j}\right)+\sum_{k=1}^{K} \pi_{k j}$. PTO $O_{k j} \in P P S$ provided that there exists $c$ $(c=1, \ldots, C)$ such that $\left(X_{j}, Y_{j}\right)+\sum_{k=1}^{K} \pi_{k j} . P T O_{k j} \in P P S_{\Gamma_{c}}$.

6. (Minimum extrapolation). PPS is the smallest set which satisfies axioms 1-5.

The meaning of axioms \#1, \#2, \#4 and \#6, are obvious. According to axiom \#3, no convex combination is allowed to be formed among the members of different local PPSs. Similarly, axiom \#5 implies that any aggregation of trade-offs is permitted only among the members of each local PPS. Now, a PPS which satisfies axioms \#1-\#6 is a pure aggregation of the local PPSs as:

$$
\begin{aligned}
& P P S=\bigcup_{c=1}^{C} P P S_{\Gamma_{c}} \quad \text { where } \quad P P S_{\Gamma_{c}}= \\
& \left\{\begin{array}{l|l}
(X, Y) \in \mathfrak{R}_{+}^{m} \times \mathfrak{R}_{+}^{s} & x_{i} \geq \sum_{j=1}^{n} \lambda_{j}^{c} x_{i j}+\sum_{j=1}^{n} \sum_{k \in \zeta_{c}} \lambda_{j}^{c} \pi_{k j}^{c} p_{i k} x_{i j}, \quad y_{r} \leq \sum_{j=1}^{n} \lambda_{j}^{c} y_{r j}+\sum_{j=1}^{n} \sum_{k \in \zeta_{c}} \lambda_{j}^{c} \pi_{k j}^{c} q_{r k} y_{r j}, \\
\sum_{j=1}^{n} \lambda_{j}^{c}=1, \quad 0 \leq \pi_{k j}^{c} \leq a_{k}, \quad \lambda_{j}^{c} \geq 0
\end{array}\right\}
\end{aligned}
$$

If we define $\omega_{k j}^{c}=\lambda_{j}^{c} \pi_{k j}^{c}$, this representation of the overall PPS can still be simplified as follows:

$$
\begin{aligned}
P P S & =\bigcup_{c=1}^{C} P P S_{\Gamma_{c}} \quad \text { where } \quad P P S_{\Gamma_{c}}= \\
& \left\{\begin{array}{l|l}
(X, Y) \in \mathfrak{R}_{+}^{m} \times \mathfrak{R}_{+}^{s} & x_{i} \geq \sum_{j=1}^{n} \lambda_{j}^{c} x_{i j}+\sum_{j=1}^{n} \sum_{k \in \zeta_{c}} \omega_{k j}^{c} p_{i k} x_{i j}, \quad y_{r} \leq \sum_{j=1}^{n} \lambda_{j}^{c} y_{r j}+\sum_{j=1}^{n} \sum_{k \in \zeta_{c}} \omega_{k j}^{c} q_{r k} y_{r j}, \\
\sum_{j=1}^{n} \lambda_{j}^{c}=1, \quad 0 \leq \omega_{k j}^{c} \leq a_{k} \lambda_{j}^{c}, \quad \lambda_{j}^{c} \geq 0
\end{array}\right\}
\end{aligned}
$$

According to the definition of the overall PPS in (12), the mathematical formulation for the computation of the efficiency of a DMU is now straightforward. The efficiency score (e.g. the input-oriented one) of a $\mathrm{DMU}_{o}$ under evaluation can be calculated by means of the following linear programming model: 


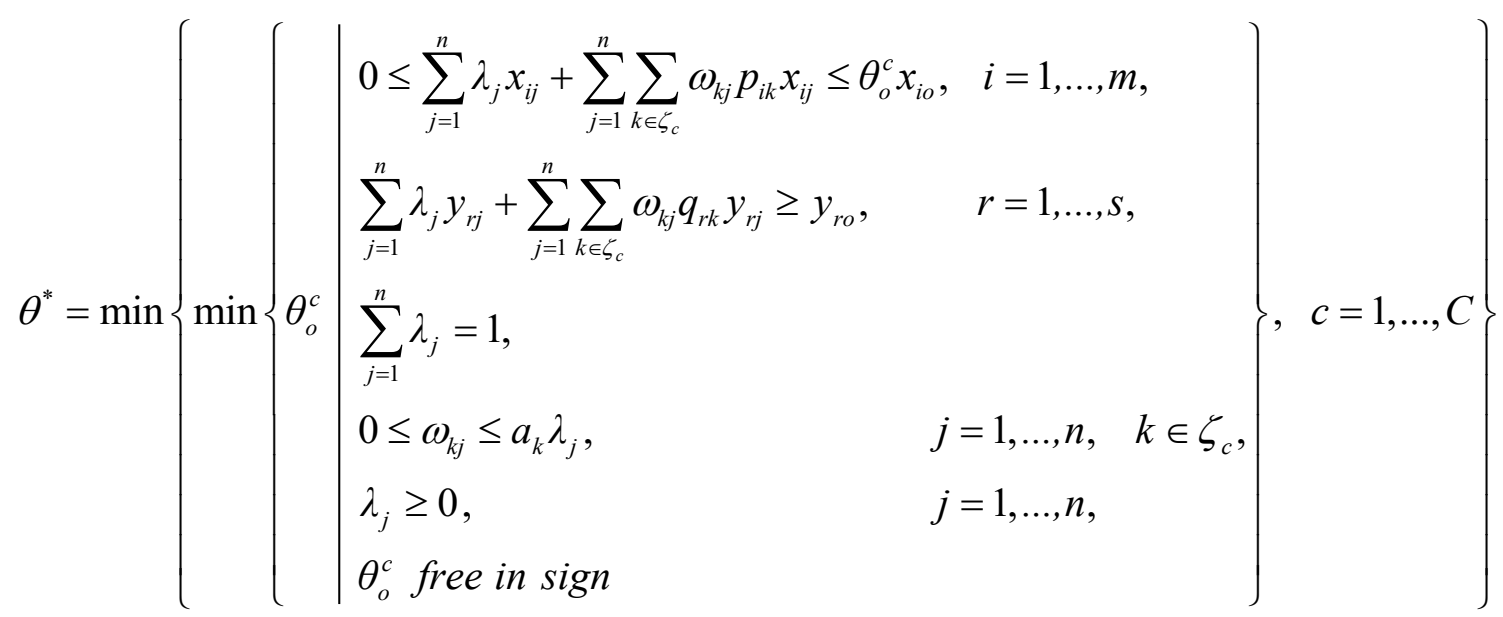

Considering (13), the problem of minimizing $\theta_{o}^{c}$ is identical with finding the smallest value among $\left\{\theta_{o}^{1^{*}}, \theta_{o}^{2^{*}}, \ldots, \theta_{o}^{C^{*}}\right\}$ in which $\theta_{o}^{c^{*}}(c=1, \ldots, C)$ can be computed by minimizing $\theta_{o}^{c}$ such that $\left(X_{o}, \theta_{o}^{c} Y_{o}\right) \in \operatorname{PPS}_{\Gamma_{c}}$. In other words, the objective function value of model (13) is determined by means of computing the optimal objective values in each local PPS separately and then finding the smallest one among them.

\section{An Empirical Illustration in the Context of Engineering Schools}

In this section, we use an example to illustrate the proposed models and the way the suggested algorithm can be applied to identify sets with consistent trade-offs. Motivated by the case of "public universities in Malaysia" given first in Podinovski and Wan Husain (2015), details of our numerical example are designed as follows. Let us assume that there exist 20 hypothetical engineering schools as DMUs in the analysis. The efficiency of the schools is to be measured by two inputs as "full time academic staff" and "total amount of research funding" (i.e. in thousand Euro) and three outputs as "number of BSc. students", "number of MSc. and PhD. students" and "research publications in reputable journals". Our data set is shown in Table 1. 


\begin{tabular}{cccccc}
\hline DMUs & Academic staff & Research & BSc. & MSc. and Ph.D. & Research publications \\
\hline 1 & 12 & 23.9 & 93 & 10 & 42 \\
2 & 77 & 7.20 & 180 & 70 & 55 \\
3 & 9 & 19.40 & 74 & 20 & 32 \\
4 & 10 & 18.70 & 84 & 21 & 33 \\
5 & 16 & 27.30 & 104 & 34 & 46 \\
6 & 15 & 12.10 & 210 & 44 & 15 \\
7 & 24 & 34.41 & 129 & 51 & 50 \\
8 & 36 & 19.90 & 212 & 66 & 31 \\
9 & 42 & 17.30 & 292 & 85 & 64 \\
10 & 16 & 9.30 & 195 & 39 & 5 \\
11 & 72 & 35.00 & 266 & 32 & 13 \\
12 & 7 & 9.70 & 71 & 5 & 11 \\
13 & 23 & 8.70 & 95 & 36 & 32 \\
14 & 15 & 15.90 & 191 & 30 & 28 \\
15 & 13 & 13.80 & 160 & 28 & 77 \\
16 & 15 & 18.13 & 178 & 32 & 47 \\
17 & 55 & 31.40 & 200 & 75 & 66 \\
18 & 66 & 11.70 & 190 & 66 & 45 \\
19 & 38 & 25.00 & 135 & 53 & \\
20 & 68 & 10.50 & 180 & 70 & \\
\hline
\end{tabular}

Table 1: Inputs and outputs of engineering schools

Following Podinovski and Wan Husain (2015), we assume that the experts believe for any university, if the number of academic staff is increased, all types of students can be increased, while the amount of research funding and research publications remain unchanged. Given the research funding unchanged, we also suppose that if the academic staff is decreased, not only the number of students but also the number of research publications decreases (for more details see Podinovski \& Wan Husain, 2015).

These assumptions can be incorporated into the analysis by means of a few proportional trade-off vectors as shown by $v_{1}-v_{5}$ in Table 2 . According to $v_{1}$, let us assume that if the academic staff is increased (or decreased) by $10 \%$ (i.e. $p_{1,1}=0.1$ ), it is possible to increase $9 \%$ (i.e. $q_{1,1}=0.09$ ) of BSc. and 7\% (i.e. $q_{1,2}=0.07$ ) of MSc. and $\mathrm{PhD}$. students, without any excessive research funding and any change in the number of research publications. With respect to $v_{2}$, it is supposed that if the number of academic staff is reduced by $8 \%$, BSc. students and as well as MSc. and PhD. students are reduced by $8 \%$ and $6.5 \%$, respectively while the number of research publications are also reduced by $6 \%$ (the level of research funding remains unchanged). In $v_{3}$, in addition to the 
academic staff reduction of $10 \%$, the amount of research funding is also assumed to be reduced by $20 \%$. According to $v_{4}$, if MSc. and $\mathrm{PhD}$. students are increased by $10 \%$, then the number of BSc. students needs to be decreased by $30 \%$ (given the other factors unchanged). Moreover, $v_{5}$ indicates that if BSc. students are increased by $40 \%$, then MSc. and $\mathrm{PhD}$. students should be decreased by $10 \%$ (given the other factors unchanged).

\begin{tabular}{cccccc}
\hline $\begin{array}{c}\text { Proportional } \\
\text { trade-off vector }\end{array}$ & $\begin{array}{c}\text { Academic } \\
\text { staff }\end{array}$ & $\begin{array}{c}\text { Research } \\
\text { funding }\end{array}$ & BSc student & $\begin{array}{c}\text { MSc. and } \\
\text { Ph.D. students }\end{array}$ & $\begin{array}{c}\text { Research } \\
\text { publications }\end{array}$ \\
\hline$v_{1}$ & $10 \%$ & 0 & $9 \%$ & $7 \%$ & $0 \%$ \\
$v_{2}$ & $-10 \%$ & 0 & $-8 \%$ & $-6.50 \%$ & $-6 \%$ \\
$v_{3}$ & $-10 \%$ & $-20 \%$ & $-12 \%$ & $-10 \%$ & $-14 \%$ \\
$v_{4}$ & $0 \%$ & 0 & $-30 \%$ & $10 \%$ & $0 \%$ \\
$v_{5}$ & $0 \%$ & 0 & $40 \%$ & $-10 \%$ & $0 \%$ \\
\hline
\end{tabular}

Table 2: Proportional trade-off vectors

We should note that the above information regarding the assessment of the schools could be incorporated into the analysis by means of the conventional approach of absolute trade-offs. In order to also show the effect of applying absolute trade-offs and compared it to the case of proportional trade-offs, let us consider the last trade-off as an example.

According to the definition of this trade-off, a similar absolute trade-off may be defined as $v_{5}^{*}$, e.g.: if BSc. students are increased by 4 units, then MSc. and PhD. students should be decreased by 1 unit (given the other factors unchanged). Now if the schools in this system are shown by $D M U_{j}=\left(x_{1 j}, x_{2 j}, y_{1 j}, y_{2 j}, y_{3 j}\right), \quad j=1, \ldots, 20, \quad$ all the feasible points on the line $D M U_{j}+\pi v_{5}^{*}=\left(x_{1 j}, x_{2 j}, y_{1 j}, y_{2 j}, y_{3 j}\right)+\pi(0,0,4,-1,0)$ will be in the PPS. This means that if a school wishes to serve 4 more (or resp. any other scale of it: $4 \pi$ units) undergraduate students, it needs to reduce 1 (or resp. $-\pi$ units) of its graduate students (without any change in the other factors). As the scaling factor $\pi$ here indicates, any scale of this trade-offs can also be applied. For example, if a school wishes to serve $4 \pi$ more undergraduate students, it needs to reduce $\pi$ of its graduate students. This will then expand the PPS accordingly. As theoretically shown in Section 2 , this can be seen by many schools as counter-intuitive and as a source of inequity in measuring their efficiency. The reason is that this trade-off has been defined in an absolute form, which does not account for the size of the schools. Within this approach, e.g., schools \#3, \#14 and \#17 
with quite different sizes will be considered to have the same potentials to change their technologies according to this trade-off. This may be seen as an unrealistic view of the change in inputs and outputs.

In contrast to this, in the new approach, the definition of a particular trade-off is adapted with respect to the volume of the inputs and outputs. Taking $v_{5}$ in Table 2 as an example of a similar proportional trade-off of $v_{5}^{*}$, we can see that all the feasible points on the line $D M U_{j}+\pi v_{5} \otimes D M U_{j}=\left(x_{1 j}, x_{2 j}, y_{1 j}, y_{2 j}, y_{3 j}\right)+\pi\left(0,0,4 y_{1 j},-y_{2 j}, 0\right)$ will be in the PPS. This means that a particular school $D M U_{j}$ can serve additionally $4 y_{1 j}$ of its undergraduate students if it can reduce the number of its graduate students by the factor of $y_{2 j}$. As the scaling factor $\pi$ here indicates, any non-negative scale of this trade-offs can also be applied. For example, if a school wants to serve $4 \pi y_{1 j}$ more undergraduate students, it will need to reduce $\pi y_{2 j}$ of its graduate students. This shows how the definition of a particular trade-off is adapted with respect to the volume of the inputs and outputs of different schools. It also highlights the fact that a trade-off, e.g., of the form $v_{5}$ is scaled up or down by which it can be applied on a particular DMU with respect to its size. Hence, we should note that although a proportional trade-off may be defined basically between $0 \%$ and $100 \%$ (for example in the case of $v_{5} 40 \%$ and $-10 \%$ ), but an appropriate value of $\pi$ will automatically adjusts the defined percentages so that it can be applied on a particular school in the system according to its size.

We note here that the corresponding translation of all proportional trade-offs in Table 2 to a set of similar absolute trade-offs has led to infeasible results of efficiency within the approach of absolute trade-offs. The reason is that the trade-offs are recognized as inconsistent within this approach by which no feasible results can be produced. In the following, however, we show how the proportional trade-offs in Table 2 can be applied by means of the proposed method. With respect to definitions 1 and 2 given in Section 3, each trade-off alone in $\Omega=\left\{v_{1}, v_{2}, v_{3}, v_{4}, v_{5}\right\}$ passes successfully through the individual consistency checks. However, the entire set of all trade-offs together is not recognized as consistent (see also Remark 1 in Section 3) meaning that a few of these trade-offs cannot be applied simultaneously. The proposed algorithm in Section 4.2 can now be used in order to identify maximal consistent 
subsets of these trade-offs. See the graphical illustration of the levels of the tree search depicted in Fig. 6. The corresponding generated subsets of the trade-offs are also given in Table 4.

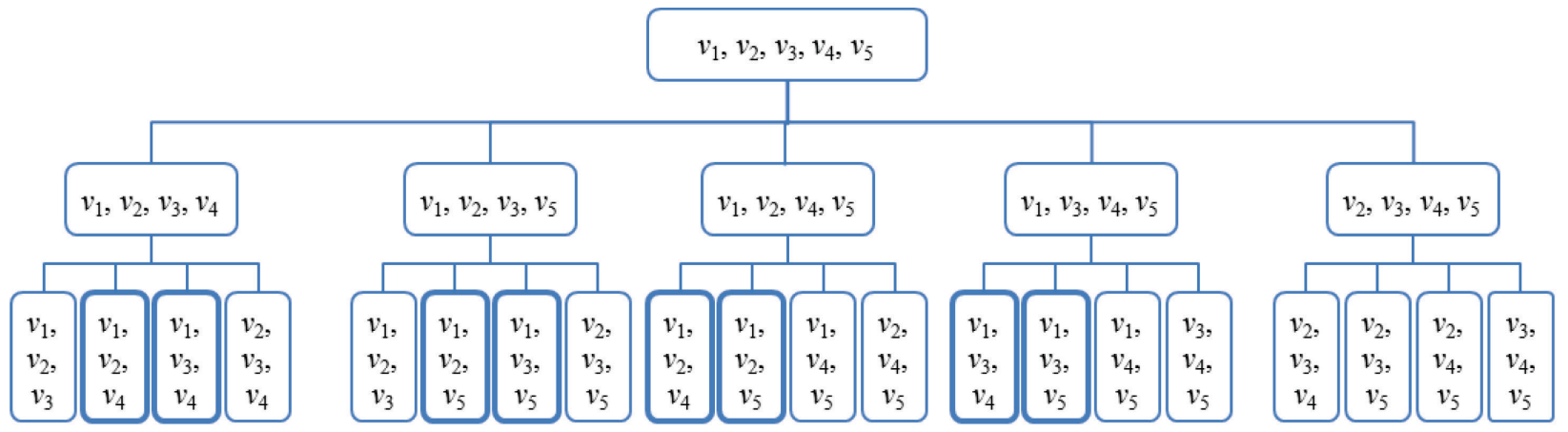

Figure 6: Recognizing maximal consistent subsets through the tree search algorithm

According to the algorithm, set $\Omega=\left\{v_{1}, v_{2}, v_{3}, v_{4}, v_{5}\right\}$ is initially located in the root of the tree, i.e. $\Pi=\left\{\Pi_{1}(=\Omega)\right\}$; see the second row of Table 3 . In the first step of the tree search (i.e. $l=1$; see the second level of the tree in Fig. 6), all subsets of $\Pi_{1}(=\Omega)$ with the cardinality of 4 are checked for consistency (i.e. see also the new $\Pi$ in the third row of Table 3). Having applied models (5) and (7) in Section 3, none of them are consistent in this level. Therefore, $\bigcup_{\forall \Gamma_{c}} \Gamma_{c}=\varnothing \neq \Omega$ and the search process including the branching procedure continues.

\begin{tabular}{lll}
\hline & \multicolumn{1}{c}{ Branches } & Consistent sets \\
\hline $\begin{array}{l}\text { Initial } \\
\text { level }\end{array}$ & $\Pi_{1}=\left\{v_{1}, v_{2}, v_{3}, v_{4}, v_{5}\right\}$ & \\
$l=1$ & $\Pi_{1}=\left\{v_{1}, v_{2}, v_{3}, v_{4}\right\}, \Pi_{2}=\left\{v_{1}, v_{2}, v_{3}, v_{5}\right\}, \Pi_{3}=\left\{v_{1}, v_{2}, v_{4}, v_{5}\right\}$, & \\
& $\Pi_{4}=\left\{v_{1}, v_{3}, v_{4}, v_{5}\right\}, \Pi_{5}=\left\{v_{2}, v_{3}, v_{4}, v_{5}\right\}$ & \\
$l=2$ & $\Pi_{1}=\left\{v_{1}, v_{2}, v_{3}\right\}, \Pi_{2}=\left\{v_{1}, v_{2}, v_{4}\right\}, \Pi_{3}=\left\{v_{1}, v_{3}, v_{4}\right\}, \Pi_{4}=\left\{v_{2}, v_{3}, v_{4}\right\}$, & $\Gamma_{1}=\left\{v_{1}, v_{2}, v_{4}\right\}, \Gamma_{2}=\left\{v_{1}, v_{3}, v_{4}\right\}$, \\
& $\Pi_{5}=\left\{v_{1}, v_{2}, v_{5}\right\}, \Pi_{6}=\left\{v_{1}, v_{3}, v_{5}\right\}, \Pi_{7}=\left\{v_{2}, v_{3}, v_{5}\right\}, \Pi_{8}=\left\{v_{1}, v_{4}, v_{5}\right\}$, & $\Gamma_{3}=\left\{v_{1}, v_{2}, v_{5}\right\}, \Gamma_{4}=\left\{v_{1}, v_{3}, v_{5}\right\}$ \\
& $\Pi_{9}=\left\{v_{2}, v_{4}, v_{5}\right\}, \Pi_{10}=\left\{v_{3}, v_{4}, v_{5}\right\}$ & \\
\hline
\end{tabular}

Table 3: Maximal consistent subsets identified by the proposed algorithm

In the second level (i.e. $l=2$ ), all subsets of each $\Pi_{1}, \Pi_{2}, \ldots, \Pi_{5}$ from the previous step - which have all a cardinality of 3 - are considered as new branches. Now the new $\Pi$ consists of ten members. Having checked the consistency for these sets, four sets are identified to be consistent 
which are withdrawn from $\Pi$ and added to $\Gamma$ (see the strikethrough sets in the fourth row of Table 3 and also branches with a bold border in Fig. 6). Now, the new set of maximal consistent sets will be $\Gamma=\left\{\Gamma_{1}, \Gamma_{2}, \ldots, \Gamma_{4}\right\}$. As $\bigcup_{c=1}^{4} \Gamma_{c}=\Omega$ then the algorithm is now terminated.

According to the results, we can observe that the pairs of 1) $v_{4}$ and $v_{5}$ and also 2) $v_{2}$ and $v_{3}$ do not appear in maximal consistent sets $\Gamma_{1}, \Gamma_{2}, \ldots, \Gamma_{4}$. With respect to Theorem $\# 1$, the pair of $v_{4}$ and $v_{5}$ creates infeasible expansion. The reason is that in model (5), a linear combination like $v_{4}+v_{5}$ exists so that the model produces a strictly positive objective function value (i.e. 0.1). This combination (i.e. $v_{4}+v_{5}$ ) shows a wrong hidden trade-off which enables output \#1 (i.e. BSc students) to arbitrarily increase on its own. Taking into account the meaning of these trade-offs, we can perceive that only one of $v_{4}$ and $v_{5}$ can be applied at a time. Because we can either just reduce the first output (allowed by $v_{4}$ but not in $v_{5}$ ) or reduce the second one (allowed by $v_{5}$ but not in $v_{4}$ ). Having a closer look at the pair of $v_{2}$ and $v_{3}$, we can similarly trace the problem back. In this case, the linear combination of $5 v_{2}+5 v_{3}$ in model (7) produces a feasible solution with strictly positive objective function value (i.e. $\rho_{2}=17.5$ ). Hence, the pair of $v_{2}$ and $v_{3}$ are identified as inconsistent, i.e. the case of free production occurs.

The efficiency of DMUs can now be evaluated by means of the overall PPS. Following the theory put forward in Section 4.3, this set is the union of the local PPSs which are formed by the already identified sets of consistent trade-offs, i.e. $P P S=\bigcup_{c=1}^{4} P P S_{\Gamma_{c}}$. The efficiency scores of DMUs evaluated by $P P S_{\Gamma_{c}}$ as well as by the overall PPS are reported in Table 4. In order to analyze the discriminatory power of our suggested model, results of the DEA model without additional trade-offs (i.e. this model is hence the standard DEA model of Banker, Charnes, \& Cooper. 1984, known as the BCC model: see Section 2) are also given in this table.

As can be seen in Table 4, the BCC model cannot discriminate DMUs well in this case. While 15 out of 20 DMUs have been reported as fully efficient, the rest has also mainly been recognized as good operators with an efficiency score above 90\%. This explains why in real applications 
additional constraints may need to be added to the analysis. This has been done in our case with a number of proportional trade-offs outlined already above. As can be taken in Table 4, the efficiency of DMUs in each local PPS is less than or equal the one reported by the BCC model. Hence, the final results - the efficiency scores by the overall PPS - which theoretically take the minimum of the efficiency scores of the local PPSs should represent a better discriminatory power than those produced by the BCC model. Evidences of this interesting and practical feature of our method can be observed by comparing the results given in the last column of Table 4 with those of the BCC model in the same table.

\begin{tabular}{ccccccc}
\hline $\mathrm{DMU}$ & $\mathrm{BCC}$ & $\theta_{o}^{1^{*}}\left(P P S_{\Gamma_{1}}\right)$ & $\theta_{o}^{2^{*}}\left(P P S_{\Gamma_{2}}\right)$ & $\theta_{o}^{3^{*}}\left(P P S_{\Gamma_{3}}\right)$ & $\theta_{o}^{4^{*}}\left(P P S_{\Gamma_{4}}\right)$ & $\theta_{o}^{*}(P P S)$ \\
\hline 1 & 1 & 0.82 & 1 & $\mathbf{0 . 7 8}$ & 1 & 0.78 \\
2 & 1 & $\mathbf{1}$ & $\mathbf{1}$ & $\mathbf{1}$ & $\mathbf{1}$ & 1 \\
3 & 1 & 0.66 & 1 & $\mathbf{0 . 6 2}$ & 1 & 0.62 \\
4 & 1 & 0.71 & 1 & $\mathbf{0 . 6 7}$ & 1 & 0.67 \\
5 & 1 & $\mathbf{0 . 7 9}$ & 1 & $\mathbf{0 . 7 9}$ & 1 & 0.79 \\
6 & 1 & 0.85 & 1 & $\mathbf{0 . 7 2}$ & 1 & 0.72 \\
7 & 1 & $\mathbf{0 . 7 4}$ & 0.95 & 0.81 & 1 & 0.74 \\
8 & 0.77 & $\mathbf{0 . 5 5}$ & 0.69 & 0.58 & 0.73 & 0.55 \\
9 & 1 & $\mathbf{1}$ & $\mathbf{1}$ & $\mathbf{1}$ & $\mathbf{1}$ & 1 \\
10 & 1 & $\mathbf{0 . 7 8}$ & 0.91 & $\mathbf{0 . 7 8}$ & 0.79 & 0.78 \\
11 & 1 & $\mathbf{1}$ & $\mathbf{1}$ & $\mathbf{1}$ & $\mathbf{1}$ & 1 \\
12 & 1 & $\mathbf{0 . 7 4}$ & 0.93 & $\mathbf{0 . 7 4}$ & $\mathbf{0 . 7 4}$ & 0.74 \\
13 & 1 & 0.83 & $\mathbf{0 . 4 9}$ & 0.83 & 0.53 & 0.49 \\
14 & 1 & 0.93 & 1 & $\mathbf{0 . 6 2}$ & 0.90 & 0.62 \\
15 & 1 & 0.98 & 1 & $\mathbf{0 . 7 2}$ & 1 & 0.72 \\
16 & 0.91 & 0.76 & 0.91 & $\mathbf{0 . 5 1}$ & 0.84 & 0.51 \\
17 & 1 & $\mathbf{0 . 8 6}$ & 0.87 & 0.91 & 0.91 & 0.86 \\
18 & 0.85 & 0.78 & $\mathbf{0 . 6 5}$ & 0.78 & 0.67 & 0.65 \\
19 & 0.96 & $\mathbf{0 . 8 8}$ & 0.94 & 0.90 & 0.96 & 0.88 \\
20 & 0.92 & 0.78 & $\mathbf{0 . 6 4}$ & 0.79 & 0.68 & 0.64 \\
\hline
\end{tabular}

Table 4: Efficiency scores obtained by the BCC model and our proposed model

Table 5 also presents some other details of the results obtained by our proposed model in (13). In addition to the efficiency scores (already also reported in Table 4 in the last column), information about reference units and intensity multipliers are given in the third and fourth columns, respectively. 


\begin{tabular}{|c|c|c|c|c|c|c|c|c|}
\hline \multirow{2}{*}{ DMU } & \multirow{2}{*}{$\theta_{o}^{*}$} & \multirow{2}{*}{ References } & \multirow{2}{*}{$\lambda_{j}^{*}$} & \multicolumn{5}{|c|}{ Trade-offs involved with the corresponding weights $\left(\pi_{k j}, \omega_{k j}\right)$} \\
\hline & & & & $v_{1}$ & $v_{2}$ & $v_{3}$ & $v_{4}$ & $v_{5}$ \\
\hline \multirow{3}{*}{1} & \multirow{3}{*}{0.78} & 2 & 0.45 & - & $(13.11,5.9)$ & - & - & - \\
\hline & & 9 & 0.16 & - & - & - & - & - \\
\hline & & 11 & 0.39 & - & $(7.19,2.8)$ & - & - & $(0.54,0.21)$ \\
\hline \multirow[t]{2}{*}{2} & \multirow{2}{*}{1} & 2 & 1 & - & - & - & - & - \\
\hline & & 2 & 0.71 & - & $(11.66,8.29)$ & - & - & - \\
\hline \multirow[t]{3}{*}{3} & \multirow[t]{3}{*}{0.62} & 9 & 0.13 & - & - & - & - & - \\
\hline & & 11 & 0.16 & - & $(6.07,0.94)$ & - & - & $(1.13,0.18)$ \\
\hline & & 2 & 0.68 & - & $(11.32,7.66)$ & - & - & - \\
\hline \multirow[t]{3}{*}{4} & \multirow[t]{3}{*}{0.67} & 9 & 0.16 & - & - & - & - & - \\
\hline & & 11 & 0.16 & - & $(7.02,1.13)$ & - & - & $(0.96,0.15)$ \\
\hline & & 2 & 0.34 & - & $(10,3.4)$ & - & $(0.35,0.12)$ & - \\
\hline \multirow[t]{2}{*}{5} & \multirow[t]{2}{*}{0.79} & 9 & 0.22 & - & - & - & - & - \\
\hline & & 11 & 0.44 & - & $(8.96,3.91)$ & - & - & - \\
\hline \multirow{2}{*}{6} & \multirow{2}{*}{0.72} & 2 & 0.69 & $(1.59,1.1)$ & $(13.9,9.56)$ & - & - & - \\
\hline & & 9 & 0.3 & $(3.21,0.98)$ & $(8.03,2.44)$ & - & - & - \\
\hline \multirow{2}{*}{7} & \multirow{2}{*}{0.74} & 9 & 0.54 & - & $(3.17,1.7)$ & - & $(0.91,0.49)$ & - \\
\hline & & 11 & 0.46 & - & $(10,4.62)$ & - & - & - \\
\hline \multirow{2}{*}{8} & \multirow{2}{*}{0.55} & 2 & 0.66 & - & $(10,6.6)$ & - & $(0.59,0.39)$ & - \\
\hline & & 9 & 0.33 & - & $(3.85,1.28)$ & - & - & - \\
\hline 9 & 1 & 9 & 1 & - & - & - & - & - \\
\hline 10 & 0.78 & 2 & 0.99 & $(5.84,5.79)$ & $(15.21,15.08)$ & - & - & - \\
\hline 11 & 1 & 11 & 1 & - & - & - & - & - \\
\hline 12 & 0.74 & 2 & 1 & - & - & - & - & - \\
\hline \multirow[t]{2}{*}{13} & 0.49 & 2 & 1 & - & - & - & - & - \\
\hline & & 2 & 0.66 & - & $(11.69,7.75)$ & - & - & - \\
\hline \multirow[t]{3}{*}{14} & 0.62 & 9 & 0.09 & - & - & - & - & $(7.7,0.7)$ \\
\hline & & 11 & 0.24 & - & $(0.49,0.12)$ & - & - & $(0.73,0.17)$ \\
\hline & & 2 & 0.72 & - & $(11.24,8.14)$ & - & - & - \\
\hline 15 & 0.72 & 9 & 0.12 & - & - & - & - & $(3.98,0.5)$ \\
\hline & & 11 & 0.15 & - & - & - & - & $(1.73,0.25)$ \\
\hline & & 2 & 0.77 & - & $(11.07,8.53)$ & - & - & - \\
\hline 16 & 0.51 & 9 & 0.02 & - & - & - & - & $(4.67,0.08)$ \\
\hline & & 11 & 0.21 & - & $(0.35,0.07)$ & - & - & $(0.12,0.03)$ \\
\hline 17 & 086 & 9 & 0.45 & - & - & - & $(2.58,1.18)$ & - \\
\hline 17 & 0.80 & 11 & 0.54 & - & $(4.39,2.38)$ & - & - & - \\
\hline 18 & 065 & 2 & 0.82 & - & - & - & - & - \\
\hline 10 & 0.05 & 9 & 0.18 & - & - & $(1.8,0.32)$ & $(4.13,0.73)$ & - \\
\hline & & 2 & 0.22 & - & $(10,2.23)$ & - & $(0.67,0.15)$ & - \\
\hline 19 & 0.88 & 9 & 0.38 & - & - & - & $(1.48,0.56)$ & - \\
\hline & & 11 & 0.4 & - & $(3.83,1.53)$ & - & - & - \\
\hline 20 & 064 & 2 & 0.90 & - & - & - & - & - \\
\hline 20 & 0.04 & 9 & 0.10 & - & - & $(5.42,0.54)$ & $(10.37,1)$ & - \\
\hline
\end{tabular}

Table 5: Efficiency scores, target DMUs and intensity variables produced by the proposed model 
As can be seen in Table 5, DMU \#2, \#9 and \#11 are the fully efficient DMUs. Therefore, our proposed model has set these DMUs as references for the inefficient DMUs. The intensity of the references for each DMU is determined by $\lambda_{j}^{*}$, s, which are also reported in the fourth column of Table 5. In columns five to nine, the pair of weights $\left(\pi_{k j}, \omega_{k j}\right)$ are given. These weights show the effect and contribution of trade-offs on the reference units. More precisely, the first element of this pair represents $\pi_{k j}=\omega_{k j} / \lambda_{j}$, which is important where the results need to be analyzed and interpreted in greater detail for the sake of target setting. In order to clarify this, take DMU \#7 as an example.

First of all, it can be seen that the efficiency score of this school (i.e. 0.74) corresponds to $P P S_{\Gamma_{1}}$ . Therefore, model (13) have selected the trade-off vectors from the consistent set $\Gamma_{1}=\left\{v_{1}, v_{2}, v_{4}\right\}$ .We can also see in Table 5 that $v_{2}$ and $v_{3}$ have never been used jointly as they have been identified as inconsistent in our approach. The same holds true for $v_{4}$ and $v_{5}$. From this Table, it can also be taken that DMUs \#9 and \#11 have been suggested as references for DMU \#7 in which:

- Effect I: Trade-offs $v_{2}$ and $v_{4}$ are applied on DMU\#9 by 3.17 and 0.91 times, respectively,

- $\quad$ Effect II: Trade-offs $v_{2}$ is applied on DMU\#11 by 10 times.

Each of these effects generate a virtual unit in the overall PPS. Taking into account now these effects, the above virtual units are combined with multipliers 0.54 and 0.46 (see $\lambda_{j}^{*}$ in the Table 5), respectively to form a specific target for DMU \#7. This is of particular importance to manages as it provides the respective school with a direction for further improvement in efficiency.

\section{Conclusions}

Proportional trade-offs have recently proposed as a method which can be used to incorporate prior views or information regarding the assessment of DMUs into relative efficiency 
measurement systems by DEA. In this approach, a trade-off is defined as a percentage change of the level of inputs/outputs. Therefore, it accounts for the size of the DMUs so that the definition of a particular trade-off is adapted with respect to the volume of the inputs and outputs. However, the incorporation of trade-offs may lead in certain cases to serious problems such as infinity or even negative efficiency scores in the results. This phenomenon has often been interpreted as a result of defining the set of trade-offs carelessly by the analyst. In this paper we have showed that this may not always be the case. We have exemplified situations in which all carefully-defined trade-offs can separately be applied without any problem. However, mathematical issues such as infinity or negative scores occur only when the trade-offs are applied all together simultaneously. Therefore, we have shown that there must exist a few tradeoffs which cannot be aggregated in "a conventional way" to expand the PPS. In other words, the existing framework by which the "trade-offs are combined mathematically" to form the PPS may cause a problem rather than the definition of the trade-offs.

Against this background, we have investigated theoretically situations in which imposing a particular trade-off can result in problems such as free production or infeasible expansion of the PPS. Furthermore, mathematical programming models have been formulated to test if "all tradeoffs" are consistent so that they can be applied simultaneously. Towards to this end, we have revisited the conventional approach of combining trade-offs. We have identified shortcomings in this approach and used it as a starting point to develop our approach of aggregating trade-offs, which avoids the above mentioned problems. As our approach requires first finding maximal consistent subsets of trade-offs to create a few local PPSs. We have also proposed an algorithm by which these sets can be identified and explained how the efficiency can be measured against a PPS which is formed by the union of local PPSs. Different properties and features of the suggested approach have been illustrated by a comprehensive numerical example.

\section{References}

Afsharian, M., Ahn, H., \& Alirezaee, M. R. (2015). Developing selective proportionality on FDH models: New insight on the proportionality axiom. International journal of information and decision sciences, 7 , 99-114. 
Alirezaee, M. R. \& Boloori, F. (2012). Proportional production trade-offs in DEA, a new developed insight into returns to scale. Asia Pacific journal of Operational Research, 29, 1317-1334.

Allen, R., Athanassopoulos, A., Dyson, R. G. \& Thanassoulis, E. (1997). Weight restrictions and value judgments in data envelopment analysis: Evolution, development and future directions. Annals of Operations Research, 73,13-34.

Angulo-Meza, L. \& Estellita-Lins, M. P. (2002). Review of methods for increasing discrimination in data envelopment analysis. Annals of Operations Research 116, 225-242.

Banker, R. D., Charnes, A. \& Cooper, W. W. (1984). Some models for estimating technical and scale inefficiencies in data envelopment analysis. Management Science, 30, 1078-1092.

Bessent, A., Bessent, W., Elam, J. \& Clark, T. (1988). Efficiency frontier determination by constraint facet analysis, Journal of Operational Research Society, 36,785 -796.

Bernroider, E. \& Stix, V. (2007). A method using weight restrictions in data envelopment analysis for ranking and validity issues in decision making. Computers and Operations Research, 34, 2637-2647.

Charnes, A., Cooper, W. W. \& Rhodes, E. (1978). Measuring the efficiency of decision making units. European Journal of Operational Research, 2, 429-444.

Charnes A., Cooper W., Huang Z. \& Sun D. (1990). Polyhedral cone-ratio DEA models with an illustrative application to large commercial banks, Journal of Econometrics, 46,73-91.

Cook, W. D., Tone, K., \& Zhu, J. (2014). Data envelopment analysis: Prior to choosing a model. Omega, $44,1-4$.

Dyson, R.G. \& Thanassoulis, E. (1988). Reducing weight flexibility in DEA, Journal of the Operations Research Society, 39, 563-576.

Hatami-Marbini, A., Rostamy-Malkhalifeh, M., Agrell, P. J., Tavana, M., \& Mohammadi, F. (2015a). Extended symmetric and asymmetric weight assignment methods in data envelopment analysis. Computers \& Industrial Engineering, 87, 621-631.

Hatami-Marbini, A., Tavana, M., Agrell, P. J., Lotfi, F. H., \& Beigi, Z. G. (2015b). A common-weights DEA model for centralized resource reduction and target setting. Computers \& Industrial Engineering, 79, 195-203.

Jahanshahloo, G. R. \& Soleimani-Damaneh, M. (2005). A note on simulating weights restrictions in DEA: An improvement of Thanassoulis and Allen's method. Computers and operations research, 32, 1037-1044.

Joro, T. \& Korhonen, P. (2015). Extension of data envelopment analysis with preference information. International series in operations research \& management science, Springer, New York. 
Khalili, M., Camanho, A. S., Portela, M. C. S. \& Alirezaee, M. R. (2010). The measurement of relative efficiency using data envelopment analysis with assurance regions that link inputs and outputs. European Journal of Operational Research, 203, 761-770.

Lotfi, F. H., Hatami-Marbini, A., Agrell, P. J., Aghayi, N., \& Gholami, K. (2013). Allocating fixed resources and setting targets using a common-weights DEA approach. Computers \& Industrial Engineering, 64, 631-640.

Mecit, E. \& Alp, I. (2013). A new proposed model of restricted data envelopment analysis by correlation coefficients. Applied mathematical modeling, 37, 3407-3425.

Nadimi, R. \& Jolai, F. (2008). Joint use of factor analysis (FA) and data envelopment analysis (DEA) for ranking of data envelopment analysis. International Journal of Mathematical, Physical and Engineering Sciences, 2, 28-222.

Olesen, O. B. \& Petersen N. C. (1991). Collinearity in data envelopment analysis: An expanded facet approach, Working paper No. 1/1991, Department of Management, Odense University, Denmark.

Podinovski, V. V. (2004a). Production trade-offs and weight restrictions in data envelopment analysis. Journal of the Operational Research Society, 55, 1311-1322.

Podinovski, V. V. (2004b). Bridging the gap between the constant and variable returns-to-scale models: Selective proportionality in data envelopment analysis. Journal of the Operational Research Society, $55,265-276$.

Podinovski, V. V. (2009). Production technologies based on combined proportionality assumptions. Journal of Productivity Analysis, 32, 21-26.

Podinovski, V. V. \& Bouzdine-Chameeva, T. (2013). Weight restrictions and free production in data envelopment analysis. Operations Research, 61, 1-12.

Podinovski, V. V. \& Bouzdine-Chameeva, T. (2015). Consistent weight restrictions in data envelopment analysis. European Journal of Operational Research, 244, 201-209

Podinovski, V. V. \& Athanassopoulos, A. D. (1998). Assessing the relative efficiency of decision making units using DEA models with weight restrictions. Journal of the Operational Research Society, 49, 500508.

Podinovski, V. V. \& Thanassoulis, E. (2007). Improving discrimination in data envelopment analysis: Some practical suggestions. Journal of Productivity Analysis, 28, 117-126.

Podinovski, V. V. \& Wan Husain, W. R. (2015). The hybrid returns-to-scale model and its extension by production trade-offs: An application to the efficiency assessment of public universities in Malaysia. Annals of Operations Research doi: 10.1007/s10479-015-1854-0. 
Premachandra, I. M. (2001). Controlling factor weights in data envelopment analysis by incorporating decision maker's value judgment: An approach based on AHP. Information and Management Sciences, $12,67-82$.

Sinuany-Stern, Z., Mehrez, A. \& Hadad, Y. (2000). An AHP/DEA methodology for ranking decision making units. International Transactions in Operational Research, 7, 109-124.

Thanassoulis, E. (1995). Assessing police forces in England and Wales using data envelopment analysis, European journal of operational research, 80, 588-607.

Thanassoulis, E., Dyson, R.G. \& Foster, M. (1987). Relative efficiency assessments using Data Envelopment Analysis: An application to data on rates departments, Journal of the Operational Research Society, 38, 397-411.

Thanassoulis, E., Portela, M. C. S. \& Allen, R. (2004). Incorporating value judgments in DEA. In: Cooper W.W., Seiford L.M., Zhu J. (eds) Handbook on data envelopment analysis. Vol 71of International Series in Operations Research \& Management science, Kluwer Academic Publishers, Boston 99-138.

Wang, Y.M., Parkan, C. \& Luo, Y. (2008). A linear programming method for generating the most favorable weights from a pairwise comparison matrix, Computers and operations research, 35, 39183930 . 